\title{
Affine Discontinuous Galerkin Method Approximation of Second-Order Linear Elliptic Equations in Divergence Form with Right-Hand Side in $L^{1}$
}

\author{
Abdeluaab Lidouh and Rachid Messaoudi \\ Department of Mathematics and Computer Science, Laboratory LACSA, Faculty of Sciences, Mohammed $1^{\text {st }}$ University, \\ BV Mohammed VI, P.O. Box 717, 60000 Oujda, Morocco
}

Correspondence should be addressed to Rachid Messaoudi; m_rachid_ens@yahoo.fr

Received 4 February 2018; Accepted 21 May 2018; Published 2 July 2018

Academic Editor: Patricia J. Y. Wong

Copyright (C) 2018 Abdeluaab Lidouh and Rachid Messaoudi. This is an open access article distributed under the Creative Commons Attribution License, which permits unrestricted use, distribution, and reproduction in any medium, provided the original work is properly cited.

We consider the standard affine discontinuous Galerkin method approximation of the second-order linear elliptic equation in divergence form with coefficients in $L^{\infty}(\Omega)$ and the right-hand side belongs to $L^{1}(\Omega)$; we extend the results where the case of linear finite elements approximation is considered. We prove that the unique solution of the discrete problem converges in $W_{0}^{1, q}(\Omega)$ for every $q$ with $1 \leq q<d /(d-1)(d=2$ or $d=3)$ to the unique renormalized solution of the problem. Statements and proofs remain valid in our case, which permits obtaining a weaker result when the right-hand side is a bounded Radon measure and, when the coefficients are smooth, an error estimate in $W_{0}^{1, q}(\Omega)$ when the right-hand side $f$ belongs to $L^{r}(\Omega)$ verifying $T_{k}(f) \in H^{1}(\Omega)$ for every $k>0$, for some $r>1$.

\section{Introduction}

In this work we consider, in dimension $d=2$ or 3 , the $\mathbb{P}_{1}$ discontinuous Galerkin (dG) method approximation of the Dirichlet problem

$$
\begin{aligned}
-\operatorname{div}(A \nabla u)=f & \text { in } \Omega, \\
u=0 & \text { on } \partial \Omega,
\end{aligned}
$$

where $\Omega$ is an open bounded set of $\mathbb{R}^{d}, A$ is a coercive matrix with coefficients in $L^{\infty}(\Omega)$, and $f$ belongs to $L^{1}(\Omega)$.

The solution of (1) does not belong to $H_{0}^{1}(\Omega)$ for a general right-hand side in $L^{1}(\Omega)$. Actually, in order to correctly define the solution of (1), one has to consider a specific framework, the concept of renormalized (or equivalently entropy) solution (see for example [1,2]). These definitions allow one to prove that in this new sense problem (1) is wellposed in the terminology of Hadamard.
For this problem the standard $\mathbb{P}_{1}$-nonconforming finite elements approximation, related to a triangulation $\mathscr{T}_{h}$ of $\Omega$, namely,

Find $u_{h} \in V_{h}$,

$$
\forall v_{h} \in V_{h},
$$

$$
a_{h}^{\text {swip }}\left(u_{h}, v_{h}\right)=\int_{\Omega} f v_{h} d x \text {, }
$$

where

$$
\begin{aligned}
V_{h} & =\left\{v_{h} \in L^{2}(\Omega): \forall T \in \mathscr{T}_{h},\left.v_{h}\right|_{T} \in \mathbb{P}_{1}[T], \forall F\right. \\
& \left.\in \mathscr{F}_{h}, \int_{F}\left[v_{h}\right]=0\right\}
\end{aligned}
$$

with the discrete bilinear form $a_{h}^{\text {swip }}$ yet to be designed, has a unique solution, since the right-hand side (2) $\int_{\Omega} f v_{h} \mathrm{~d} x$ is 
correctly defined for $f \in L^{1}(\Omega)$ and the bilinear form $a_{h}^{\text {swip }}$ is consistent.

Using the ideas which are at the root of the SWIP (Symmetric Weighted Interior Penalty) method, in the case $f \in L^{2}(\Omega)$, D. A. Di Pietro and A. Ern have proved, in [3], that the unique solution $u_{h}$ of (2) converges to the unique solution $u$ of (1) in the following sense:

$$
\begin{gathered}
u_{h} \longrightarrow u \quad \text { strongly in } L^{2}(\Omega), \\
\nabla_{h} u_{h} \longrightarrow \nabla u \quad \text { strongly in }\left[L^{2}(\Omega)\right]^{d}, \\
\left|u_{h}\right|_{J} \longrightarrow 0, \\
\text { when } h \longrightarrow 0,
\end{gathered}
$$

with the broken gradient $\nabla_{h}$ and the jump seminorm $|\cdot|_{J}$ yet to be designed.

To do that, the authors in [3] assume that the family of triangulations $\mathscr{T}_{h}$ belong to an admissible mesh sequence in the sense of 17 and is compatible with the partition $\mathscr{P}_{\Omega}$ (see Assumption 3).

The framework in this paper is the same as in [3]. The unique difference here is that $f \in L^{1}(\Omega)$ is considered instead of $f \in L^{2}(\Omega)$; and we ourselves focus on the two cases $d=2$ and $d=3$. The same convergence results are proved.

Notations. In the present work, $\Omega$ denotes an open bounded subset of $\mathbb{R}^{d}$ with $d=2$ or $d=3$. A particular case is the case where $\Omega$ is an open bounded polyhedron. We use the notation $A v w$ for the scalar product of the vector $A v$ by the vector $w$ (which is often denoted by ${ }^{t} w \cdot A v$ ). For a measurable set $S \subset \Omega$, we denote by $|S|$ the measure of $S$ and by $S^{c}$ the complement $\Omega \backslash S$ of $S$.

For $1<p<+\infty$ and $m \geq 0$, we have

$$
\begin{aligned}
& H^{m}\left(\mathscr{T}_{h}\right):=\left\{v \in L^{2}(\Omega) / \forall T \in \mathscr{T}_{h}, v_{\left.\right|_{T}} \in H^{m}(T)\right\}, \\
& W^{m, p}\left(\mathscr{T}_{h}\right) \\
& \quad:=\left\{v \in L^{p}(\Omega) / \forall T \in \mathscr{T}_{h}, v_{\left.\right|_{T}} \in W^{m, p}(T)\right\} .
\end{aligned}
$$

We define also the following function spaces:

$$
\begin{aligned}
& H(\operatorname{div}, \Omega):=\left\{\tau \in\left[L^{2}(\Omega)\right]^{d} / \nabla \cdot \tau \in L^{2}(\Omega)\right\}, \\
& H\left(\operatorname{div}, \mathscr{T}_{h}\right) \\
& \quad:=\left\{\tau \in\left[L^{2}(\Omega)\right]^{d} / \forall T \in \mathscr{T}_{h}, \tau_{\left.\right|_{T}} \in H(\operatorname{div}, T)\right\} .
\end{aligned}
$$

For $k \geq 0$, we define the broken polynomial space

$$
\mathbb{P}_{d}^{k}\left(\mathscr{T}_{h}\right):=\left\{v \in L^{2}(\Omega) / \forall T \in \mathscr{T}_{h}, v_{\left.\right|_{T}} \in \mathbb{P}_{d}^{k}(T)\right\},
$$

with polynomial degree $\mathrm{k} \geq 1$.

In that case, $\mathbb{P}_{d}^{1}\left(\mathscr{T}_{h}\right) \subset H^{1}\left(\mathscr{T}_{h}\right)$, which leads us to define the broken gradient $\nabla_{h}: H^{1}\left(\mathscr{T}_{h}\right) \rightarrow\left[L^{2}(\Omega)\right]^{d}$ such that, $\forall v \in$ $H^{1}\left(\mathscr{T}_{h}\right)$,

$$
\forall T \in \mathscr{T}_{h}:\left.\quad\left(\nabla_{h} v\right)\right|_{T}:=\nabla\left(v_{\left.\right|_{T}}\right)
$$

and the broken divergence operator $\nabla_{h}: H\left(\operatorname{div}, \mathscr{T}_{h}\right) \rightarrow$ $\left[L^{2}(\Omega)\right]^{d}$ such that, $\forall v \in H\left(\operatorname{div}, \mathscr{T}_{h}\right)$,

$$
\forall T \in \mathscr{T}_{h}:\left.\quad\left(\nabla_{h} \cdot v\right)\right|_{T}:=\nabla \cdot\left(v_{\left.\right|_{T}}\right) .
$$

Moreover, for any mesh element $T \in \mathscr{T}_{h}$, we denote

$$
\begin{aligned}
& \forall T \in \mathscr{T}_{h}, \quad \mathscr{F}_{T}:=\left\{F \in \mathscr{F}_{h} / F \in \partial T\right\}, \\
& \forall F \in \mathscr{F}_{h}, \quad \mathscr{T}_{F}:=\left\{T \in \mathscr{T}_{h} / F \in \partial T\right\} .
\end{aligned}
$$

And for a scalar-valued function $\mathrm{v}$ defined on $\Omega$ (which can admit two possible traces) the average of $\mathrm{v}$ is defined as

$$
\begin{aligned}
& \{v\}_{F}(x):=\frac{1}{2}\left(v_{\left.\right|_{T_{1}}}(x)+v_{\left.\right|_{T_{2}}}(x)\right) ; \\
& \text { if } F \in \mathscr{F}_{h}^{i} \text { such that } F \subset \partial T_{1} \cap \partial T_{2}, \\
& \{v\}_{F}(x):=v_{\left.\right|_{T}}(x), \\
& \text { if } F \in \mathscr{F}_{h}^{b} \text { such that } F \subset \partial T \cap \partial \Omega,
\end{aligned}
$$

and the jump of $\mathrm{v}$ as

$$
\begin{aligned}
& {[v]_{F}(x):=v_{\left.\right|_{T_{1}}}(x)-v_{\left.\right|_{T_{2}}}(x),} \\
& \text { if } F \in \mathscr{F}_{h}^{i} \text { such that } F \subset \partial T_{1} \cap \partial T_{2}, \\
& {[v]_{F}(x):=v_{\left.\right|_{T}}(x),} \\
& \text { if } F \in \mathscr{F}_{h}^{b} \text { such that } F \subset \partial T \cap \partial \Omega .
\end{aligned}
$$

For any face $\mathrm{F} \in \mathscr{F}_{h}$ and for any integer $l \geq 0$, we define the (local) lifting operator $r_{F, A}^{l}: L^{2}(F) \rightarrow \mathbb{P}_{d}^{l}\left(\mathscr{T}_{h}\right)$ as follows. For all $\varphi \in L^{2}(F)$,

$$
\begin{aligned}
\int_{\Omega} A r_{F, A}^{l}(\varphi) \cdot \tau_{h}=\int_{F}\left\{A \tau_{h}\right\} \cdot n_{F} \varphi & \\
\forall & \forall \tau_{h} \in\left[\mathbb{P}_{d}^{l}\left(\mathscr{T}_{h}\right)\right]^{d},
\end{aligned}
$$

and for any function $v \in H^{1}\left(\mathscr{T}_{h}\right)$, we define the (global) lifting of its interface and boundary jumps as

$$
R_{h, A}^{l}([v])=\sum_{F \in \mathscr{F}_{h}} r_{F, A}^{l}([v]) \in\left[\mathbb{P}_{d}^{l}\left(\mathscr{T}_{h}\right)\right]^{d} .
$$

We also introduce the normal diffusion coefficient to one face $F$ as

$$
\begin{aligned}
& \forall F \in \mathscr{F}_{h}^{i}, \quad F=\partial T_{1} \cap \partial T_{2}: \\
& A_{i}:=\left(A_{\left.\right|_{T_{i}}} n_{F}\right) \cdot n_{F}, \quad i \in\{1,2\},
\end{aligned}
$$

the diffusion-dependent penalty parameter (harmonic average of normal diffusion) as

$$
\gamma_{A, F}:= \begin{cases}\frac{2 A_{1} A_{2}}{A_{1}+A_{2}} & \text { if } F \subset \partial T_{1} \cap \partial T_{2}, \\ \left(A_{I_{T}} n\right) \cdot n & \text { if } F \subset \partial T \cap \partial \Omega,\end{cases}
$$


the weighted average operator for all $F \in \mathscr{F}_{h}^{i}$ such that $F \subset$ $\partial T_{1} \cap \partial T_{2}$ as

$$
\begin{aligned}
& \omega_{T_{1}, F}:=\frac{A_{2}}{A_{1}+A_{2}}, \\
& \omega_{T_{2}, F}:=\frac{A_{1}}{A_{1}+A_{2}},
\end{aligned}
$$

the weighted average operator for all $F \in \mathscr{F}_{h}^{i}$ and for a.e. $x \in$ $F$ as

$$
\{v\}_{\omega, F}(x):=\omega_{T_{1}, F} v_{\left.\right|_{T_{1}}}(x)+\omega_{T_{2}, F} v_{\left.\right|_{T_{2}}}(x),
$$

on boundary faces $\mathrm{F} \in \mathscr{F}_{h}^{b}$ such that $\mathrm{F} \subset \partial T \cap \partial \Omega$, we set

$$
\{v\}_{\omega, F}(x):=v_{\left.\right|_{T}}(x),
$$

and the skew-weighted average operator for all $F \in \mathscr{F}_{h}^{i}$ and for a.e. $x \in F$, as

$$
\{v\}_{\bar{\omega}, F}(x):=\omega_{T_{2}, F} v_{\left.\right|_{T_{1}}}(x)+\omega_{T_{1}, F} v_{\left.\right|_{T_{2}}}(x) .
$$
[3])

The SWIP bilinear form is defined by (see Lemma 4.47 in

$$
\begin{gathered}
\mathrm{a}_{h}^{\text {swip }}(v, w):=\underbrace{\int_{\Omega} A \nabla_{h} v \nabla_{h} w d x}_{\text {consistency term }}+\underbrace{\sum_{F \in \mathscr{F}_{h}} \eta \frac{\gamma_{A, F}}{h_{F}} \int_{F}[v, w]}_{\text {penalty term }} \\
-\underbrace{\sum_{F \in \mathscr{F}_{h}} \int_{F}\left(\left\{A \nabla_{h} v\right\}_{\bar{\omega}} \cdot n_{F}[w]+\left\{A \nabla_{h} w\right\}_{\omega} \cdot n_{F}[v]\right),}_{\text {symmetry term }}
\end{gathered}
$$

where the quantity $\eta>0$ denotes a user-dependent penalty parameter which is independent of the diffusion coefficient.

And the SWIP norms are defined by

$$
\begin{aligned}
\|v\|_{\text {swip }}:= & \left(\left\|A^{1 / 2} \nabla_{h} v\right\|_{\left[L^{2}(\Omega)\right]^{d}}^{2}+|v|_{A, J}^{2}\right)^{1 / 2}, \\
\forall q \geq 1: & \\
& \|v\|_{\text {swip,q }}:=\left(\left\|A^{1 / 2} \nabla_{h} v\right\|_{\left[L^{q}(\Omega)\right]^{d}}^{q}+|v|_{J, A, q}^{q}\right)^{1 / q},
\end{aligned}
$$

with the diffusion-dependent jump seminorms

$$
\begin{aligned}
|v|_{J, A}^{2}:=\sum_{F \in \mathscr{F}_{h}} \frac{\gamma_{A, F}}{h_{F}}\|[v]\|_{L^{2}(F)}^{2}, \\
\forall q \geq 1: \quad|v|_{J, A, q}^{q}:=\sum_{F \in \mathscr{F}_{h}} \frac{\gamma_{A, F}}{h_{F}^{q-1}}\|[v]\|_{L^{q}(F)}^{q} .
\end{aligned}
$$

The discrete Galerkin norm is defined by

$$
\|v\|_{d G}:=\left(\left\|\nabla_{h} v\right\|_{\left[L^{2}(\Omega)\right]^{d}}^{2}+|v|_{J}^{2}\right)^{1 / 2}
$$

with the jump seminorm

$$
|v|_{J}^{2}:=\sum_{F \in \mathscr{F}_{h}} \frac{1}{h_{F}}\|[v]\|_{L^{2}(F)}^{2} .
$$

For every $r$ with $1<r<+\infty$, we denote by $L^{r, \infty}(\Omega)$ the Marcinkiewicz space whose norm is defined by

$$
\|v\|_{L^{r, \infty}(\Omega)}=\sup _{\lambda>0} \lambda|\{x \in \Omega:|v(x)| \geq \lambda\}|^{1 / r} .
$$

For every real number $k>0$ we define the truncation $T_{k}: \mathbb{R} \rightarrow \mathbb{R}$ by

$$
T_{k}(s)=\max (-k, \min (k, s))= \begin{cases}s & \text { if }|s| \leq k \\ k \frac{s}{|s|} & \text { if }|s| \geq k\end{cases}
$$

For every $d$ - simplex $T$ in $\mathbb{R}^{d}$, we adopt the following notations:

(i) $a_{i, T}, i=0, \ldots, d$, denote the vertices of $T$.

(ii) $m_{i, T}, i=0, \ldots, d$, denote the centers of the faces $F_{i} \in$ $T$.

(iii) $\lambda_{i, T}, i=0, \ldots, d$, designate the barycentric coordinates with respect to the $a_{i, T}$ 's.

(iv) for every $x \in \mathbb{R}^{d}$ we put

$$
\varphi_{i, T}(x):=1-d \lambda_{i, T}(x) \quad \text { for } i=0, \ldots, d,
$$

where $\left(\varphi_{i, T}\right)_{0 \leq i_{\leq} d}$ are the $\mathbb{P}_{1}$ shape functions related to $T$; it is known that

$$
\begin{aligned}
\varphi_{i} & \in L^{2}\left(\Omega_{h}\right), \\
\varphi_{\left.i\right|_{T}} & \in \mathbb{P}_{1}[T],
\end{aligned}
$$

$$
\forall i \in\{0,1, \ldots, d\}, \forall T \in \mathscr{T}_{h} \text {, }
$$

$\varphi_{\left.i\right|_{F_{i}}}=1$,

$$
\varphi_{i}\left(a_{i}\right)=1-d,
$$

$\varphi_{i}\left(a_{j}\right)=1$ such that $j \neq i$,

$\frac{1}{\left|F_{j}\right|} \int_{F_{j}} \varphi_{i}=\varphi_{i}\left(m_{j}\right)=\delta_{i, j}$

$\sum_{i=0}^{d} \varphi_{i, T}(x)=1, \quad$ for every $\mathrm{x} \in \mathbb{R}^{d}$,

with $\Omega_{h}:=\bigcup\left\{T, T \in \mathscr{T}_{h}\right\} \subset \bar{\Omega}$. 
(v) If $\mathrm{N}$ designate the number of all interior centers $m_{i}$ of faces $\mathrm{F}$ in $\mathscr{T}_{h}$ we define the interpolation operator $\Pi_{h}$ and the truncated interpolation operator $I_{h}^{k}$ by

$$
\begin{aligned}
\forall v & \in L^{2}(\bar{\Omega}) \text { avec } \int_{F}\left[v_{h}\right]=0, \\
\Pi_{h}(v) & \in V_{h} \\
\Pi_{h}(v) & :=\sum_{1 \leq i \leq N} \alpha_{i}^{v} \varphi_{i} \\
I_{h}^{k}(v) & :=\sum_{1 \leq i \leq N} T_{k}\left(\alpha_{i}^{v}\right) \varphi_{i}
\end{aligned}
$$

with $\alpha_{i}^{v}=\Pi_{h}(v)\left(m_{i}\right)=\left(1 /\left|F_{i}\right|\right) \int_{F_{i}} \Pi_{h}(v):=$ $\left(1 /\left|F_{i}\right|\right) \int_{F_{i}} v$.

(vi) Finally, we define the $N \times N$ stiffness matrix $Q=\left(Q_{i j}\right)$; namely,

$Q_{i, j}=a_{h}^{\text {swip }}\left(\varphi_{i}, \varphi_{j}\right)$ for $i, j$ in $\{1,2, \ldots, N\}$.

As in [4], the main assumption of the present paper is that $Q$ is a diagonally dominant matrix; namely,

$$
\forall i \in\{1,2, \ldots, N\}: \quad Q_{i i}-\sum_{\substack{1 \leq j \leq N \\ j \neq i}}\left|Q_{i j}\right| \geq 0
$$

\section{Statement of the Main Result}

We consider a matrix $A$ such that

$$
\begin{gathered}
A \in L^{\infty}(\Omega)^{d \times d}, \\
\text { a.e } x \in \Omega: \forall \xi \in \mathbb{R}^{d}: A(x) \xi \xi \geq \alpha|\xi|^{2},
\end{gathered}
$$

for some $\alpha>0$, and a right-hand side $f$ such that

$$
f \in L^{1}(\Omega) \text {. }
$$

A function $u$ is the renormalized solution of the problem (1) if $u$ satisfies

$$
\begin{aligned}
& u \in L^{1}(\Omega), \\
& \forall k>0, \quad T_{k}(u) \in H_{0}^{1}(\Omega), \\
& \lim _{k \rightarrow \infty} \frac{1}{k} \int_{\Omega}\left|\nabla T_{k}(u)\right|^{2} \mathrm{~d} x=0, \\
& \forall k>0, \forall S \in \mathscr{C}_{c}^{1}(\mathbb{R}) \text { with supp }(S) \subset[-k, k], \\
& \forall v \in H_{0}^{1}(\Omega) \cap L^{\infty}(\Omega), \\
& \int_{\Omega} A \nabla T_{k}(u) \nabla v S(u) \mathrm{d} x \\
& \quad+\int_{\Omega} A \nabla T_{k}(u) \nabla T_{k}(u) S^{\prime}(u) v \mathrm{~d} x \\
& \quad=\int_{\Omega} f S(u) v \mathrm{~d} x .
\end{aligned}
$$

It is known (see $[1,5])$ that when $f$ belongs to $L^{1}(\Omega) \cap$ $H^{-1}(\Omega)$, the usual weak solution of (1), namely,

$$
\begin{array}{r}
u \in H_{0}^{1}(\Omega), \\
\forall v \in H_{0}^{1}(\Omega), \\
\int_{\Omega} A \nabla v \nabla u \mathrm{~d} x=\int_{\Omega} f v \mathrm{~d} x,
\end{array}
$$

is a renormalized solution of (1) and conversely the main interest of definition of renormalized solution is the following existence, uniqueness, and continuity theorem (see $[1,4]$ ).

Theorem 1. Assume that $A$ and $f$ satisfy (33), (34), and (35). Then there exists a renormalized solution of (1). This solution is unique. Moreover this unique solution belongs to $W_{0}^{1, q}(\Omega)$ for every $q$ with $1 \leq q<d /(d-1)$. It depends continuously on the right-hand side $f$ in the following sense: if $f^{\mathcal{E}}$ is a sequence which satisfies

$$
f^{\varepsilon} \longrightarrow f \text { strongly in } L^{1}(\Omega)
$$

when $\varepsilon$ tends to zero, then the sequence $u^{\varepsilon}$ of the renormalized solutions of (1) for the right-hand sides $f^{\varepsilon}$ satisfies for every $k>$ 0 and for every $q$ with $1 \leq q<d /(d-1)$

$$
\begin{gathered}
T_{k}\left(u^{\varepsilon}\right) \longrightarrow T_{k}(u) \quad \text { strongly in } H_{0}^{1}(\Omega), \\
u^{\varepsilon} \longrightarrow u \text { strongly in } W_{0}^{1, q}(\Omega),
\end{gathered}
$$

when $\varepsilon$ tends to zero, where $u$ is the renormalized solution of (1) for the right-hand side $f$. Finally, if $f_{1}$ and $f_{2}$ belong to $L^{1}(\Omega)$, and if $u_{1}$ and $u_{2}$ are the renormalized solutions of (1) for the right-hand sides $f_{1}$ and $f_{2}$, then, for every $k>0$, the function $T_{k}\left(u_{1}-u_{2}\right)$ belongs to $H_{0}^{1}(\Omega)$ and for every $q$ with $1 \leq q<$ $d /(d-1)$ one has

$$
\begin{aligned}
\alpha\left\|T_{k}\left(u_{1}-u_{2}\right)\right\|_{H_{0}^{1}(\Omega)}^{2} \leq k\left\|f_{1}-f_{2}\right\|_{L^{1}(\Omega)}, & \\
& \alpha\left\|u_{1}-u_{2}\right\|_{W_{0}^{1, q}(\Omega)} \leq C(d,|\Omega|, q)\left\|f_{1}-f_{2}\right\|_{L^{1}(\Omega)},
\end{aligned}
$$

Where the constant $C(d,|\Omega|, q)$ only depends on $d,|\Omega|$, and $q$.

Remark 2. Throughout all this paper, we denote by $C\left(p_{1}, p_{2}, p_{3}, ..\right)$ any real constant which only depends on the parameters $p_{1}, p_{2}$, and $p_{3} \ldots$. We can use the same notation for different constants. 
Now we consider a family of triangulations $\mathscr{T}_{h}$ satisfying for each $h>0$ the following assumption:

the triangulation $\mathscr{T}_{h}$ is made of a finite number of closed

$d$-simplices $T$ (namely triangles when $d=2$ and tetrahedra

$$
\text { when } d=3 \text { ) such that : }
$$

(i) $\Omega_{h}=\bigcup\left\{T: T \in \mathscr{T}_{h}\right\} \subset \bar{\Omega}$,

(ii) for every compact set $K$ with $K \subset \Omega$, there exists

$h_{0}(K)>0$ such that $K \subset \Omega_{h}$ for every $h$ with $h<h_{0}(K)$,

(iii) for $\left(T_{1}, T_{2}\right) \in \mathscr{T}_{h}^{2}$ with $T_{1} \neq T_{2}$, one has $\left|T_{1} \cap T_{2}\right|=0$,

(iv) every face of every $T$ of $\mathscr{T}_{h}$ is either a subset of $\partial \Omega_{h}$, or a face of another $T^{\prime}$ of $\mathscr{T}_{h}$.

Note that because of (iv) the triangulations are conforming. A particular case is the case where $\Omega$ is a polyhedron of $\mathbb{R}^{d}$, and where $\Omega_{h}$ coincides with $\Omega$ for every $h$.

In practice, the diffusion coefficient (i.e., matrix A) has more regularity than just belonging to $L^{\infty}(\Omega)$. Henceforth, we make the following assumption (assumption 4.43 [3]):

there is a partition $P_{\Omega}$

$$
:=\left\{\Omega_{i}\right\}_{1 \leq i \leq N_{\Omega}} \text { of } \Omega \text { such that : }
$$

(i) Each $\Omega_{i}, \quad 1 \leq i \leq N_{\Omega}$, is apolyhedron;

(ii) The restriction of $\mathrm{A}$ to each $\Omega_{i}, 1 \leq i$

$$
\leq N_{\Omega} \text {, is constant. }
$$

An important assumption on the mesh sequence $\mathscr{T}_{h}:=$ $\left(\mathscr{T}_{\mathscr{C}}\right)_{h \in \mathscr{H}}$ is its compatibility with the partition $P_{\Omega}$ in the following sense (assumption 4.45 [3]).

Assumption 3 (mesh compatibility). We suppose that the admissible mesh sequence $\mathscr{T}_{\mathscr{H}}$ is such that, for each $h \in \mathscr{H}$, each $T \in \mathscr{T}_{h}$ is a subset of only one set $\Omega_{i}$ of the partition $P_{\Omega}$. In this situation, the meshes are said to be compatible with the partition $P_{\Omega}$.

For every $T \in \mathscr{T}_{h}$, we denote by $h_{T}$ the diameter of $T$ and by $\rho_{T}$ the diameter of the ball inscribed in $T$. We set

$$
h=\sup _{T \in \mathscr{T}_{h}} h_{T}
$$

and we assume that $h$ tends to zero.

We also assume that the family of triangulations $\mathscr{T}_{h}$ is regular in the sense of P. G. Ciarlet [6]; namely, there exists a constant $\sigma$ such that

$$
\forall h, \forall T \in \mathscr{T}_{h}, \quad \frac{h_{T}}{\rho_{T}} \leq \sigma .
$$

For every triangulation $\mathscr{T}_{h}$, we consider the discrete problem:

$$
\begin{gathered}
\text { Find } u_{h} \in V_{h}, \\
\forall v_{h} \in V_{h}, \\
a_{h}^{\text {swip }}\left(u_{h}, v_{h}\right)=\int_{\Omega} f v_{h} d x .
\end{gathered}
$$

Note that the right-hand side of (48) makes sense since $f \in L^{1}(\Omega)$ and $V_{h} \subset L^{\infty}(\Omega)$. The discrete bilinear forme $a_{h}^{\text {swip }}$ is consistent and coercive (see (128)) on $V_{h}$, so a straightforward consequence of the Lax-Milgram Lemma is that the discrete problem (48) is well-posed. The solution $u_{h}$ of (48) exists and is unique.

As in [4], the main result of this paper is the following.

Theorem 4. Assume that $A, f$, and $\mathscr{T}_{h}$ satisfy (33), (34), (35), (44), (46), (47), and (32). Then the unique solution $u_{h}$ of (48) satisfies for every $k>0$ and for every $q$ with $1 \leq q<d /(d-1)$

$$
\begin{aligned}
u_{h} & \longrightarrow u \quad \text { strongly in } L^{q}(\Omega), \\
\nabla_{h} u_{h} & \longrightarrow \nabla u \quad \text { strongly in }\left[L^{q}(\Omega)\right]^{d}, \\
\left|u_{h}\right|_{J, A, q} & \longrightarrow 0, \\
I_{h}^{k}\left(u_{h}\right) & \longrightarrow T_{k}(u) \quad \text { strongly in } L^{2}(\Omega), \\
\nabla_{h} I_{h}^{k}\left(u_{h}\right) & \longrightarrow \nabla T_{k}(u) \quad \text { strongly in }\left[L^{2}(\Omega)\right]^{d}, \\
\left|I_{h}^{k}\left(u_{h}\right)\right|_{J, A} & \longrightarrow 0,
\end{aligned}
$$

when $h$ tends to zero, where $u$ is the unique renormalized solution of (1).

This theorem will be proved in Section 4, using the tools that we will prepare in Section 3. In Section 5, we will explain why the results of [4] when $f$ is a bounded Radon measure remain valid in our case. In Section 6 we also show that if we assume in addition that $T_{k}(f) \in H^{1}(\Omega)$ for every $k>0$, we obtain for smooth solutions an $O\left(h^{4(1-1 / r)}\right)$ error estimate in \|\|$_{\text {swip, }}$-norm (Section 6.1), and for Low-Regularity solutions an $O\left(h^{4 \alpha_{p}(1-1 / r)}\right)$ error estimate in \|\|$_{\text {swip, } q}$-norm (Section 6.2). Finally, in Section 7 we show that in the case where A is the identity matrix, condition (32) remains satisfied when every inner angle of every d-simplex of $\mathscr{T}_{h}$ is acute.

\section{Tools}

We are going to prove Theorem 4 in several steps. We begin by proving the following result which is a piecewise $\mathbb{P}_{1}$ variant of a result of L. Boccardo \& T. Gallouët $[2,5]$.

Theorem 5. Assume that $v_{h} \in V_{h}$ satisfies

$$
\forall k>0, \quad \int_{\Omega}\left|\nabla_{h} I_{h}^{k}\left(v_{h}\right)\right|^{2} \mathrm{~d} x \leq k M
$$


for some $M>0$. Then, for every $q$ with $1 \leq q<d /(d-1)$,

$$
\left\|v_{h}\right\|_{\text {swip,q }} \leq C_{2}\left(d,|\Omega|, q, \sigma,\|A\|_{L^{\infty}(\Omega)^{d \times d}}\right) M
$$

where the constant $C_{2}(d,|\Omega|, q)$ only depends on $d,|\Omega|$, and $q$.

As in [4], to prove Theorem 5, we use the following lemmas.

Lemma 6. Under assumption (47), for all $T \in \mathscr{T}_{h}$ and all $F \in$ $\mathscr{F}_{T}$, one has

$$
h_{F}|F| \leq 2 \sigma^{d}|T|
$$

Proof. Indeed, let $T \in \mathscr{T}_{h}$ and $F \in \mathscr{F}_{T}$, so

$$
h_{F}|F| \leq h_{T}^{d}
$$

and by (47) one has

$$
h_{F}|F| \leq \sigma^{d} \rho_{T}^{d}
$$

which combined with the fact that

$$
|T| \geq c \rho_{T}^{d}
$$

where $c=\Pi / 4$ in 2D, and $c=\Pi / 6$ in 3D, implies (52).

Lemma 7. Under assumption (47), for every $q$ such that $1 \leq q$, the following bound holds for any $v_{h} \in V_{h}$ :

$$
|v|_{J, A, q}^{q} \leq C\left(q, \sigma, d,\|A\|_{L^{\infty}(\Omega)^{d \times d}}\right)\left\|\nabla_{h} v\right\|_{L^{q}(\Omega)} .
$$

Proof. For every $T \in \mathscr{T}_{h}$, we denote by $\left(\nabla_{h} v\right)_{T}$ the (constant) gradient of the restriction of $v$ to $T$. With this notation, using the continuity of $v$ across any $F$ in $\mathscr{F}_{T}$ at the mass center $x_{F}$ of any internal $F$, the fact that $v$ vanishes at the mass center $x_{F}$ of any external $F$, and the known inequality

$$
\forall q \geq 1, \quad(|a|+|b|)^{q} \leq 2^{q-1}\left(|a|^{q}+|b|^{q}\right)
$$

and using (52) we get

$$
\begin{aligned}
& |v|_{, A, q}^{q}=\sum_{F \in \mathscr{F}_{h}^{i}} \frac{\gamma_{A, F}}{h_{F}^{q-1}} \int_{F}[v]^{q} d x+\sum_{F \in \mathscr{F}_{h}^{b}} \frac{\gamma_{A, F}}{h_{F}^{q-1}} \int_{F}[v]^{q} d x \\
& \leq \sum_{\substack{F \in \mathscr{F}_{h}^{i} \\
F=T_{1} \cap T_{2}}} \frac{\gamma_{A, F}}{h_{F}^{q-1}} \\
& \cdot \int_{F}\left(\left(\left(\nabla_{h} v\right)_{T_{1}}-\left(\nabla_{h} v\right)_{T_{2}}\right) \cdot\left(x-x_{F}\right)\right)^{q} d x \\
& +\sum_{\substack{F \in \mathscr{F}_{h}^{b} \\
F \in \mathscr{F}_{T}}} \int_{F} \frac{\gamma_{A, F}}{h_{F}^{q-1}}\left(\left(\nabla_{h} v\right)_{T} \cdot\left(x-x_{F}\right)\right)^{q} d x \\
& \leq \sum_{\substack{F \in \mathscr{F}_{h}^{i} \\
F=T_{1} \cap T_{2}}} 2^{q} \min \left(\lambda_{T_{1}, F}, \lambda_{T_{2}, F}\right) h_{F}|F| \\
& \cdot\left(\left|\left(\nabla_{h} v\right)_{T_{1}}\right|^{q}+\left|\left(\nabla_{h} v\right)_{T_{2}}\right|^{q}\right)+\sum_{\substack{F \in \mathscr{F}_{h}^{b} \\
F \in \mathscr{F}_{T}}} \lambda_{T, F} h_{F}|F| \\
& \cdot\left|\left(\nabla_{h} v\right)_{T}\right|^{q} \leq 2 \sigma^{d}\|A\|_{L^{\infty}(\Omega)^{d \times d}} \sum_{\substack{F \in \mathscr{F}_{h}^{i} \\
F=T_{1} \cap T_{2}}} 2^{q} \\
& \cdot\left(\left|T_{1}\right|\left|\left(\nabla_{h} v\right)_{T_{1}}\right|^{q}+\left|T_{2}\right|\left|\left(\nabla_{h} v\right)_{T_{2}}\right|^{q}\right) \\
& +2 \sigma^{d}\|A\|_{L^{\infty}(\Omega)^{d \times d}} \sum_{\substack{F \in \mathscr{F}_{h}^{b} \\
F \in \mathscr{F}_{T}}}|T|\left|\left(\nabla_{h} v\right)_{T}\right|^{q} \\
& \leq 2^{q+3} \sigma^{d}\|A\|_{L^{\infty}(\Omega)} \sum_{T \in \mathscr{T}_{h}}|T|\left|\left(\nabla_{h} v\right)_{T}\right|^{q},
\end{aligned}
$$

which is (56) with $C\left(q, \sigma, d,\|A\|_{L^{\infty}(\Omega)^{d \times d}}\right)=2^{q+3} \sigma^{d}\|A\|_{L^{\infty}(\Omega)^{d \times d}}$.

Lemma 8. Let $v_{h} \in V_{h}$ and let $k>0$. Iffor some $T \in \mathscr{T}_{h}$ there exists $y \in T$ with $\left|v_{h}(y)\right| \geq k$, then there exists a $d$-simplex $S \subset T$ with $|S|=c(d)|T|$ such that

$$
\forall x \in S: \quad\left|I_{h}^{k}\left(v_{h}(x)\right)\right| \geq \frac{k}{2},
$$

and the strictly positive constant $c(d)$ only depends on $d$.

Proof. Let $T$ be a $d$-simplex from the triangulation $\mathscr{T}_{h}, v_{h} \in$ $V_{h}$, and $k>0$, such that $\sup _{T}\left|v_{h}\right| \geq k$. Consider $w_{h}=I_{h}^{k}\left(v_{h}\right) \epsilon$ $\mathbb{P}_{1}(T)$.

(i) If $\max _{m_{i} \in T}\left|v_{h}\right|<k$, thus

$$
\begin{aligned}
\forall i & \in\{0,1, \ldots, d\}, \\
T_{k}\left(\alpha_{i}^{v_{h}}\right) & =T_{k}\left(v_{h}\left(m_{i}\right)\right)=v_{h}\left(m_{i}\right)=\alpha_{i}^{v_{h}}
\end{aligned}
$$

so $w_{h}=v_{h}$, and $\exists y \in T$ such that $\left|w_{h}(y)\right| \geq k$.

(ii) If $\max _{m_{i} \in T}\left|v_{h}\right| \geq k$, thus $\exists m_{i} \in T$, such that $\left|v_{h}\left(m_{i}\right)\right| \geq$ $k$ so $\left|T_{k}\left(v_{h}\left(m_{i}\right)\right)\right|=k$, and $\left|w_{h}\left(m_{i}\right)\right|=T_{k}\left(\alpha_{i}^{v_{h}}\right)=k$. 
In both cases, there exists an element $y$ in $T$ such that $\left|w_{h}(y)\right| \geq k$.

But $w_{h} \in \mathbb{P}_{1}(T)$, so

$$
w_{h}(y)-w_{h}(x)=\nabla w_{h}(y-x)
$$

In other words

$$
\begin{aligned}
\forall i \neq j \neq k \text { dans }\{0,1, \ldots, d\}, \\
\nabla \lambda_{i}\left(a_{i}-a_{k}\right)=1, \\
\nabla \lambda_{i}\left(a_{j}-a_{k}\right)=0 .
\end{aligned}
$$

and since

$$
y-x=\sum_{k=1}^{d}\left(\lambda_{k}(y)-\lambda_{k}(x)\right)\left(a_{k}-a_{0}\right),
$$

one obtains

$$
\begin{gathered}
\nabla w_{h}=-d \sum_{i=0}^{d} T_{k}\left(\alpha_{i}^{v_{h}}\right) \nabla \lambda_{i}, \\
\nabla w_{h}\left(a_{k}-a_{o}\right)=-d\left(T_{k}\left(\alpha_{k}^{v_{h}}\right)-T_{k}\left(\alpha_{0}^{v_{h}}\right)\right),
\end{gathered}
$$

so

$$
\left|\nabla w_{h}\left(a_{k}-a_{o}\right)\right| \leq 2 k d
$$

and

$$
\left|w_{h}(y)-w_{h}(x)\right| \leq 2 d \sum_{k=1}^{d}\left|\lambda_{k}(y)-\lambda_{k}(x)\right| \leq \frac{k}{2},
$$

as soon as

$$
\sum_{k=1}^{d}\left|\lambda_{k}(y)-\lambda_{k}(x)\right| \leq \frac{1}{4 d} .
$$

For this purpose we define the $d$-simplex $S=\left(y-a_{0}\right)+S_{0}$ such that

$$
S_{0}=\left\{x, \lambda_{0} \geq 1-\frac{1}{4 d}=\frac{4 d-1}{4 d}\right\}
$$

so

$$
\left|w_{h}(x)\right| \geq\left|w_{h}(y)\right|-\left|w_{h}(y)-w_{h}(x)\right| \geq k-\frac{k}{2}=\frac{k}{2},
$$

and to estimate the measure of $S$, it is clear to verify first that $|S|=\left|S_{0}\right|$. Let $\widehat{T}$ be the reference unit $d$-simplex with vertices $\widehat{a}_{0}=0, \widehat{a}_{1}=e_{1}, \ldots, \widehat{a}_{d}=e_{d}$, where $\left\{e_{i}, i=1, \ldots, d\right\}$ is the canonical basis of $\mathbb{R}^{d}$. Let $F_{T}$ be the invertible affine mapping that $\operatorname{maps} F_{T}(\widehat{T})=T$ onto $T$ and set $\widehat{S}=F_{T}^{-1}(S)$.

Since $F_{T}$ is affine, it is easy to check that $\hat{\lambda}_{i}=F_{T}^{-1} \circ \lambda_{i, T}$ for $i=0,1, \ldots, d$ are the barycentric coordinates with respect to the $\hat{a}_{i}$ 's and that

$$
\begin{aligned}
\widehat{S} & =\left\{\widehat{x} \in \widehat{T}: \widehat{\lambda_{0}}(\widehat{x}) \geq \frac{4 d-1}{4 d}\right\}, \\
|S| & =\frac{|\widehat{S}|}{|\widehat{T}|}|T|=C(d)|T|,
\end{aligned}
$$

where $c(d)=|\widehat{S}| /|\widehat{T}|$ is a constant that depends only on $d$. This proves the result.
Lemma 9. Assume that $v_{h} \in V_{h}$ satisfies (50), then

$$
\left|B_{k}\left(v_{h}\right)\right| \leq C\left(d,|\Omega|, 2^{*}\right)\left(\frac{M}{k}\right)^{2^{*} / 2},
$$

for every $k>0$, where $2^{*}=2 d /(d-2)=6$ if $d=3$ and $2^{*}$ is any real number with $2^{*} \geq 1$ if $d=2 ; B_{k}\left(v_{h}\right)$ is defined by

$$
B_{k}\left(v_{h}\right)=\bigcup\left\{T \in \mathscr{T}_{h}: \max _{T}\left|v_{h}\right| \geq k\right\}
$$

and $C\left(d,|\Omega|, 2^{*}\right)$ is a constant depending only on $d, 2^{*}, \theta_{0}$, and $|\Omega|$.

Proof. Discrete Sobolev's theorem (see theorem 5.3 in [3]) asserts that

$$
\forall v \in V_{h}, \quad\|v\|_{L^{2^{*}}(\Omega)} \leq \sigma_{2,2^{*}}(d,|\Omega|)\|v\|_{d G},
$$

and we will also need (56) with $q=2$

$$
\forall v \in V_{h}, \quad|v|_{J}^{2} \leq C(\sigma, d)\left\|\nabla_{h} v\right\|_{L^{2}(\Omega)} .
$$

Here $d=2$ or $d=3$ so $2^{*}=2 d /(d-2)=6$ if $d=3$ and $2^{*}$ can be any real number with $1 \leq 2^{*}$.

Fix $k>0$. If $T \subset B_{k}\left(v_{h}\right)$, from Lemma 8, we know that there exists $S \subset T$, with $|S|=c(d)|T|$ and

$$
\forall x \in S, \quad\left|I_{h}^{k}\left(v_{h}(x)\right)\right| \geq \frac{k}{2} .
$$

Therefore

$$
\begin{aligned}
\int_{T}\left|I_{h}^{k}\left(v_{h}(x)\right)\right|^{2^{*}} \mathrm{~d} x & \geq \int_{S}\left|I_{h}^{k}\left(v_{h}(x)\right)\right|^{2^{*}} \mathrm{~d} x \\
& \geq\left(\frac{k}{2}\right)^{2^{*}}|S|=c(d)|T|\left(\frac{k}{2}\right)^{2^{*}} .
\end{aligned}
$$

Hence

$$
\begin{aligned}
\left|B_{k}\left(v_{h}\right)\right| & =\sum_{T \subset B_{k}\left(v_{h}\right)}|T| \\
& \leq \sum_{T \subset B_{k}\left(v_{h}\right)} \frac{1}{c(d)}\left(\frac{2}{k}\right)^{2^{*}} \int_{T}\left|I_{h}^{k}\left(v_{h}(x)\right)\right|^{2^{*}} \mathrm{~d} x \\
& \leq \frac{1}{c(d)}\left(\frac{2}{k}\right)^{2^{*}} \int_{\Omega}\left|I_{h}^{k}\left(v_{h}(x)\right)\right|^{2^{*}} \mathrm{~d} x .
\end{aligned}
$$

Combining with (73) and (74) one has 


$$
\left|B_{k}\left(v_{h}\right)\right| \leq \frac{\left(2 \sigma_{2,2^{*}}(d,|\Omega|)\right)^{2^{*}}(1+C(\sigma, d))^{2^{*} / 2}}{c(d) k^{2^{*}}}\left(\int_{\Omega}\left|\nabla_{h} I_{h}^{k}\left(v_{h}(x)\right)\right|^{2} \mathrm{~d} x\right)^{2^{*} / 2} .
$$

Finally using (50), we obtain

$$
\begin{aligned}
& \left|B_{k}\left(v_{h}\right)\right| \\
& \quad \leq \frac{\left(2 \sigma_{2,2^{*}}(d,|\Omega|)\right)^{2^{*}}(1+C(\sigma, d))^{2^{*} / 2}}{c(d)}\left(\frac{M}{k}\right)^{2^{*} / 2},
\end{aligned}
$$

which is (71) with

$$
\begin{aligned}
C & \left(d,|\Omega|, 2^{*}, \sigma,\|A\|_{L^{\infty}(\Omega)^{d \times d}}\right) \\
& =\frac{\left(2 \sigma_{2,2^{*}}(d,|\Omega|)\right)^{2^{*}}}{C(d)}(1+C(\sigma, d))^{2^{*} / 2} .
\end{aligned}
$$

Proof (proof of Theorem 5; see [4]). Fix $q$ with $1 \leq q<d /(d-$ 1). Take $r=\left(2 \times 2^{*}\right) /\left(2+2^{*}\right)=3 / 2$ in the case $d=3$, and verify $\left(2 \times 2^{*}\right) /\left(2+2^{*}\right)>q$, in the case $d=2$.

The embedding inequality $\left(L^{q}(\Omega) \hookrightarrow L^{r, \infty}(\Omega)\right)$ writes

$$
\left\|\left|\nabla_{h} v_{h}\right|\right\|_{L^{q}(\Omega)} \leq C(q, r,|\Omega|)\left\|\left|\nabla v_{h}\right|\right\|_{L^{r, \infty}(\Omega)},
$$

and by (56) one can have

$$
\begin{aligned}
& \forall v \in W_{0}^{1, q}\left(\mathscr{T}_{h}\right), \\
&|v|_{J, A, q}^{q} \leq C\left(q, \sigma, d,\|A\|_{L^{\infty}(\Omega)^{d \times d}}\right)\left\|\nabla_{h} v\right\|_{L^{q}(\Omega)} .
\end{aligned}
$$

So to prove Theorem 5, it suffices to estimate

$$
\left\|\left|\nabla v_{h}\right|\right\|_{L^{r, \infty}(\Omega)}=\sup _{\lambda>0}\left|\bigcup_{T \in T_{h}}\left\{x \in T:\left|\nabla_{h} v_{h}\right| \geq \lambda\right\}\right|^{1 / r} .
$$

So let $\lambda>0$. For every $k>0$, we can write

$$
\begin{aligned}
& \left|\bigcup_{T \in T_{h}}\left\{x \in T:\left|\nabla_{h} v_{h}\right| \geq \lambda\right\}\right| \\
& \leq\left|\bigcup_{T \in T_{h}}\left\{x \in T:\left|\nabla_{h} v_{h}\right| \geq \lambda\right\} \cap B_{k}\left(v_{h}\right)\right| \\
& +\left|\bigcup_{T \in T_{h}}\left\{x \in T:\left|\nabla_{h} v_{h}\right| \geq \lambda\right\} \cap\left[B_{k}\left(v_{h}\right)\right]^{c}\right| \\
& \leq\left|B_{k}\left(v_{h}\right)\right| \\
& +\left|\bigcup_{T \in T_{h}}\left\{x \in T:\left|\nabla_{h} v_{h}\right| \geq \lambda\right\} \cap\left[B_{k}\left(v_{h}\right)\right]^{c}\right| .
\end{aligned}
$$

But $\left\{x \in \Omega:\left|\nabla v_{h}(x)\right| \geq \lambda\right\} \cap\left[B_{k}\left(v_{h}\right)\right]^{c}$ coincides, up to a set of measure zero, with

$$
\begin{aligned}
& \bigcup_{T \in T_{h}}\left(\left\{x \in T:\left|\nabla_{h} v_{h}\right| \geq \lambda\right\}\right. \\
& \left.\cap\left\{T \in \mathscr{T}_{h}: \max _{T}\left|v_{h}\right|<k\right\}\right) .
\end{aligned}
$$

On the other hand, if $\max _{T}\left|v_{h}\right|<k$, then $I_{h}^{k}\left(v_{h}\right)_{\left.\right|_{T}}=v_{\left.h\right|_{T}}$, so

$$
\begin{aligned}
& \left|\bigcup_{T \in T_{h}}\left\{x \in T:\left|\nabla_{h} v_{h}\right| \geq \lambda\right\} \cap\left[B_{k}\left(v_{h}\right)\right]^{c}\right| \\
& \leq\left|\bigcup_{T \in T_{h}}\left\{x \in T:\left|\nabla \Pi_{h} T_{k}\left(v_{h}\right)\right| \geq \lambda\right\}\right| \\
& \quad \leq \frac{1}{\lambda^{2}} \int_{\Omega}\left|\nabla_{h} \Pi_{h}\left(T_{k}\left(v_{h}\right)\right)\right|^{2} d x .
\end{aligned}
$$

By the use of (50), one has

$$
\left|\bigcup_{T \in T_{h}}\left\{x \in T:\left|\nabla_{h} v_{h}\right| \geq \lambda\right\} \cap\left[B_{k}\left(v_{h}\right)\right]^{c}\right| \leq \frac{k M}{\lambda^{2}} .
$$

We fix $k=\lambda^{2-r} M^{r-1}$ to have $k M / \lambda^{2}=(M / \lambda)^{r}$; using (71) we obtain

$$
\begin{aligned}
& \left|\bigcup_{T \in T_{h}}\left\{x \in T:\left|\nabla_{h} v_{h}\right| \geq \lambda\right\}\right| \\
& \leq C\left(d,|\Omega|, 2^{*}\right)\left(\frac{M}{k}\right)^{2^{*} / 2}+\left(\frac{M}{\lambda}\right)^{r},
\end{aligned}
$$

so, for $r=\left(2 \times 2^{*}\right) /\left(2+2^{*}\right)$ to have $(M / k)^{2^{*} / 2}=(M / \lambda)^{r}$, in both cases $d=2$ or $d=3$, one has

$$
\begin{aligned}
& \left|\bigcup_{T \in T_{h}}\left\{x \in T:\left|\nabla_{h} v_{h}\right| \geq \lambda\right\}\right| \\
& \leq\left(C\left(d,|\Omega|, 2^{*}\right)+1\right)\left(\frac{M}{\lambda}\right)^{r},
\end{aligned}
$$

and then

$$
\lambda\left|\bigcup_{T \in T_{h}}\left\{x \in T:\left|\nabla_{h} v_{h}\right| \geq \lambda\right\}\right|^{1 / r} \leq C(d,|\Omega|, q) M,
$$

for every $\lambda>0$, which finishes the proof. 
Lemma 10. Let $v \in V_{h}$. For every $s, k$ with $0 \leq s<k$, the set $B_{k, s}(v)$, defined by

$$
B_{k, s}(v)=\bigcup_{T \in T_{h}}\left\{T \in \mathscr{T}_{h}: \min _{T}|v| \leq s, \max _{T}|v| \geq k\right\},
$$

satisfies

$$
\left|B_{k, s}(v)\right| \leq \frac{h^{2}}{(k-s)^{2}} \int_{\Omega}\left|\nabla_{h}(v)\right|^{2} \mathrm{~d} x .
$$

Proof. If $T \in B_{k, s}(v), \max _{T}|v|=|v(a)| \geq k$, and $\min _{T}|v|=$ $|v(b)| \leq s$, so

$$
\begin{aligned}
k-s & \leq|v(a)|-|v(b)| \leq|v(a)-v(b)| \\
& \leq|\nabla(v)||a-b| .
\end{aligned}
$$

Therefore

$$
1 \leq|\nabla(v)|^{2} \frac{|a-b|^{2}}{(k-s)^{2}}
$$

Hence

$$
\begin{aligned}
\left|B_{k, s}(v)\right| & =\sum_{T \in B_{k, s}(v)}|T|=\sum_{T \in B_{k, s}(v)} \int_{T} 1 d x \\
& \leq \sum_{T \in B_{k, s}(v)} \int_{T}|\nabla(v)|^{2} \frac{|a-b|^{2}}{(k-s)^{2}} d x \\
& \leq \frac{h^{2}}{(k-s)^{2}} \int_{\Omega}\left|\nabla_{h}(v)\right|^{2} d x .
\end{aligned}
$$

The estimate (92) follows.

Lemma 11. Let $v_{h} \in V_{h}$ and $0 \leq s<k$, then the set $\widetilde{B}_{k, s}\left(v_{h}\right)$, defined by

$$
\begin{aligned}
& \widetilde{B}_{k, s}\left(v_{h}\right) \\
& =\bigcup\left\{T \in \mathscr{T}_{h}: \min _{m_{i} \in T}\left|v_{h}\right| \leq s, \max _{T}\left|v_{h}\right| \geq k\right\},
\end{aligned}
$$

satisfies

$$
\left|\widetilde{B}_{k, s}\left(v_{h}\right)\right| \leq \frac{h^{2}}{(k-s)^{2}} \int_{\Omega}\left|\nabla_{h} I_{h}^{k}\left(v_{h}\right)\right|^{2} \mathrm{~d} x .
$$

Proof. Indeed, if $T \subset \widetilde{B}_{k, s}\left(v_{h}\right)$ then there are two possibilities:

(i) If $\max _{m_{i} \in T}\left|v_{h}\right| \leq k$ so $I_{h}^{k}\left(v_{h}\right)_{\left.\right|_{T}}=v_{\left.h\right|_{T}}$.

(ii) If $\max _{m_{i} \in T}\left|v_{h}\right| \geq k$ so $\max _{T}\left|I_{h}^{k}\left(v_{h}\right)\right| \geq k$ and obviously $\min _{T}\left|I_{h}^{k}\left(v_{h}\right)\right| \leq s$.

In the two cases $T \subset B_{k, s}\left(I_{h}^{k}\left(v_{h}\right)\right)$. The estimate (97) follows.

Remark 12. It is then clear that, under hypothesis (50),

$$
\begin{array}{r}
\left|\widetilde{B}_{k, s}\left(v_{h}\right)\right| \underset{h \rightarrow 0}{\longrightarrow} 0, \\
\left|B_{k, s}\left(I_{h}^{k}\left(v_{h}\right)\right)\right| \underset{h \rightarrow 0}{\longrightarrow} 0 .
\end{array}
$$

In addition, one has the following.
Proposition 13. Let $v_{h} \in V_{h}$ and $0 \leq s<k$. If $v_{h}$ satisfies (50), then

$$
\left|B_{k, s}\left(v_{h}\right)\right| \underset{h \rightarrow 0}{\longrightarrow} 0 .
$$

Proof. Fix $k>0$ and $s>0, s<k$. For $h>0$ such that $1 / h \geq k$, we can write

$$
\begin{aligned}
B_{k, s}\left(v_{h}\right)= & \left(B_{k, s}\left(v_{h}\right) \cap B_{1 / h}\left(v_{h}\right)\right) \\
& \cup\left(B_{k, s}\left(v_{h}\right) \cap\left[B_{1 / h}\left(v_{h}\right)\right]^{c}\right) .
\end{aligned}
$$

On the one hand, with $2^{*}=6$ in (71), one has

$$
\left|B_{k, s}\left(v_{h}\right) \cap B_{1 / h}\left(v_{h}\right)\right| \leq\left|B_{1 / h}\left(v_{h}\right)\right| \leq C(|\Omega|) h^{3} .
$$

On the other hand,

$$
B_{k, s}\left(v_{h}\right) \cap\left[B_{1 / h}\left(v_{h}\right)\right]^{c} \subset B_{k, s}\left(I_{h}^{1 / h}\left(v_{h}\right)\right) .
$$

Indeed, if $x \in B_{k, s}\left(v_{h}\right) \cap\left[B_{1 / h}\left(v_{h}\right)\right]^{c}$ and $T \in \mathscr{T}_{h}$ such that $x \in T$, then $\max _{T}\left|v_{h}\right| \geq k, \min _{T}\left|v_{h}\right| \leq s$, and, for every $y$ in $T,\left|v_{h}(y)\right| \leq 1 / h$, which means $\left.I_{h}^{1 / h}\left(v_{h}\right)\right|_{T}=\left.v_{h}\right|_{T}$, and $T \epsilon$ $B_{k, s}\left(I_{h}^{1 / h}\left(v_{h}\right)\right)$.

Therefore, with Lemma 10, and (50), one has

$$
\begin{aligned}
\left|B_{k, s}\left(v_{h}\right) \cap B_{1 / h}^{c}\left(v_{h}\right)\right| & \leq\left|B_{k, s}\left(I_{h}^{1 / h}\left(v_{h}\right)\right)\right| \\
& \leq \frac{h^{2}}{(k-s)^{2}} \int_{\Omega}\left|\nabla_{h} I_{h}^{1 / h}\left(v_{h}\right)\right|^{2} \mathrm{~d} x \\
& \leq \frac{h}{(k-s)^{2}} M .
\end{aligned}
$$
(103).

The convergence (99) is then a consequence of (101) and

Lemma 14. Let $v_{h} \in V_{h}$. For every s and every $k$ with $0<s<$ $k$, one has

$$
\begin{gathered}
\left\{x \in \Omega: T_{s}\left(I_{h}^{k}\left(v_{h}\right)\right) \neq T_{s}\left(v_{h}(x)\right)\right\} \\
\subset B_{k, s}\left(v_{h}\right) \cup B_{k, s}\left(I_{h}^{k}\left(v_{h}\right)\right) .
\end{gathered}
$$

Proof. Let $x \in \Omega$ such that $T_{s}\left(I_{h}^{k}\left(v_{h}(x)\right)\right) \neq T_{s}\left(v_{h}(x)\right)$, and $T \in \mathscr{T}_{h}$ with $x \in T$. It is easily checked that $\left.I_{h}^{k}\left(v_{h}\right)\right|_{T} \neq$ $\left.v_{h}\right|_{T}, \max _{\widetilde{T}}\left|v_{h}\right| \geq k$, and $\max _{\widetilde{T}}\left|I_{h}^{k}\left(v_{h}\right)\right| \geq k$. So there are three possibilities.

(i) $\left|I_{h}^{k}\left(v_{h}(x)\right)\right| \geq s$ and $\left|v_{h}(x)\right|<s$, and then $T \subset B_{k, s}\left(v_{h}\right)$,

(ii) $\left|I_{h}^{k}\left(v_{h}(x)\right)\right|<s$ and $\left|v_{h}(x)\right| \geq s$, and then $T \subset$ $B_{k, s}\left(I_{h}^{k}\left(v_{h}\right)\right)$,

(iii) $\left|I_{h}^{k}\left(v_{h}(x)\right)\right|<s$ and $\left|v_{h}(x)\right|<s$, and then

$$
T \subset B_{k, s}\left(I_{h}^{k}\left(v_{h}\right)\right) \cap B_{k, s}\left(v_{h}\right) .
$$

In all cases $x \in T \subset B_{k, s}\left(I_{h}^{k}\left(v_{h}\right)\right) \cup B_{k, s}\left(v_{h}\right)$, and (104) follows. 
Proposition 15. Assume that $v_{h} \in V_{h}$ satisfies (50). Then, for every $k>0$, one has

$$
I_{h}^{k}\left(v_{h}\right)-T_{k}\left(v_{h}\right) \longrightarrow 0 \text { in measure, }
$$

when $h$ tends to zero.

Proof. Fix $k>0$ and $\varepsilon>0$ such that $\varepsilon<k$ and consider

$$
I_{\varepsilon}=\left\{x \in \Omega:\left|I_{h}^{k}\left(v_{h}(x)\right)-T_{k}\left(v_{h}(x)\right)\right| \geq \varepsilon\right\} .
$$

Let $x \in I_{\varepsilon}$ and $T \in \mathscr{T}_{h}$ with $x \in T$. It is easily checked that

$$
I_{h}^{k}\left(v_{h}\right)_{\left.\right|_{T}} \neq T_{k}\left(v_{h}\right)_{\left.\right|_{T}},
$$

which implies that $\max _{T}\left|v_{h}\right|>k$. So there are four possibilities.

(i) $v_{h}$ changes sign in $T$; then, by continuity,

$$
\left.T \subset B_{k, s}\left(v_{h}\right) \quad \text { for every } s \in\right] 0, k[\text {. }
$$

(ii) $I_{h}^{k}\left(v_{h}\right)$ changes sign in $T$; then

$$
\left.T \subset B_{k, s}\left(I_{h}^{k}\left(v_{h}\right)\right) \quad \text { for every } s \in\right] 0, k[\text {. }
$$

(iii) $\max _{m_{i} \in T}\left|v_{h}\right| \leq k$ and $v_{\left.h\right|_{T}} \geq 0$ (or $v_{\left.h\right|_{T}} \leq 0$ ) so $I_{h}^{k}\left(v_{h}\right)_{\mid T}=v_{h \mid T}$.

(a) If $v_{\left.h\right|_{T}} \geq 0$, then $v_{h}(x) \geq k+\varepsilon$ and $T \subset$ $B_{k+\varepsilon, k}\left(I_{h}^{k}\left(v_{h}\right)\right)$.

(b) If $v_{\left.h\right|_{T}} \leq 0$, then $v_{h}(x) \leq-k-\varepsilon$ and $T \subset$ $B_{k+\varepsilon, k}\left(I_{h}^{k}\left(v_{h}\right)\right)$.

(iv) $\max _{m_{i} \in T}\left|v_{h}\right|>k$ and $v_{\left.h\right|_{T}} \geq 0$ (or $v_{\left.h\right|_{T}} \leq 0$ ). So

$$
\begin{aligned}
& \left|I_{h}^{k}\left(v_{h}(x)\right)-T_{k}\left(v_{h}(x)\right)\right| \\
& \quad=|| I_{h}^{k}\left(v_{h}(x)\right)|-| T_{k}\left(v_{h}(x)\right)|| .
\end{aligned}
$$

(a) If $\left|I_{h}^{k}\left(v_{h}(x)\right)\right|-\left|T_{k}\left(v_{h}(x)\right)\right| \geq \varepsilon$. There are three possibilities:

case 1: $\quad\left|v_{h}(x)\right| \geq k$, so $\left|I_{h}^{k}\left(v_{h}(x)\right)\right| \geq k+\varepsilon$ and

$$
T \subset B_{k+\varepsilon, k}\left(I_{h}^{k}\left(v_{h}\right)\right) ;
$$

case 2: $\quad\left|v_{h}(x)\right|<k-\varepsilon / 2$, so $T \subset B_{k, k-\varepsilon / 2}\left(v_{h}\right)$;

case 3: $\quad k-\varepsilon / 2 \leq\left|v_{h}(x)\right|<k$, so $\left|I_{h}^{k}\left(v_{h}(x)\right)\right| \geq$ $k+\varepsilon / 2$ and

$$
T \subset B_{k+\varepsilon / 2, k}\left(I_{h}^{k}\left(v_{h}\right)\right) .
$$

(b) If $\left|T_{k}\left(v_{h}(x)\right)\right|-\left|I_{h}^{k}\left(v_{h}(x)\right)\right| \geq \varepsilon$, then

$$
\left|I_{h}^{k}\left(v_{h}(x)\right)\right| \leq\left|T_{k}\left(v_{h}(x)\right)\right|-\varepsilon,
$$

so

$$
T \subset B_{k, k-\varepsilon}\left(I_{h}^{k}\left(v_{h}\right)\right) .
$$

We can then conclude that

$$
\begin{gathered}
I_{\varepsilon} \subset B_{k+\varepsilon / 2, k}\left(\Pi_{h} T_{k}\left(v_{h}\right)\right) \cup B_{k, k-\varepsilon}\left(\Pi_{h} T_{k}\left(v_{h}\right)\right) \\
\cup B_{k, k-\varepsilon / 2}\left(v_{h}\right) .
\end{gathered}
$$

Convergence (106) is then consequence of (98) and (99).

The result and the proof of the Proposition (2.7) in [4] can be conserved without changes.

Proposition 16. Under assumption (32), one has for every $v_{h} \epsilon$ $V_{h}$ and every $k>0$

$$
a_{h}^{\text {swip }}\left(v_{h}-I_{h}^{k}\left(v_{h}\right), I_{h}^{k}\left(v_{h}\right)\right) \geq 0 .
$$

Proof (proof of Proposition 2.7 in [4]). Since

$$
\begin{gathered}
v_{h}=\sum_{i=0}^{d} \alpha_{i}^{v_{h}} \varphi_{i}, \\
I_{h}^{k}\left(v_{h}\right)=\sum_{i=0}^{d} T_{k}\left(\alpha_{i}^{v_{h}}\right) \varphi_{i},
\end{gathered}
$$

one has

$$
\begin{aligned}
a_{h}^{\text {swip }} & \left(v_{h}-I_{h}^{k}\left(v_{h}\right), I_{h}^{k}\left(v_{h}\right)\right)=\int_{\Omega} A \nabla_{h}\left(v_{h}-I_{h}^{k}\left(v_{h}\right)\right) \\
\cdot & \nabla_{h} I_{h}^{k}\left(v_{h}\right) d x \\
& -\sum_{F \in \mathscr{F}_{h}^{i}} \int_{F}\left\{A \nabla_{h}\left(v_{h}-I_{h}^{k}\left(v_{h}\right)\right)\right\}_{\bar{\omega}} \cdot n_{F}\left[I_{h}^{k}\left(v_{h}\right)\right] d x \\
& -\sum_{F \in \mathscr{F}_{h}^{i}} \int_{F}\left\{A \nabla_{h} I_{h}^{k}\left(v_{h}\right)\right\}_{\omega} \cdot n_{F}\left[v_{h}-I_{h}^{k}\left(v_{h}\right)\right] d x \\
& +\sum_{F \in \mathscr{F}_{h}^{i}} \int_{F} \frac{\gamma_{A, F}}{h_{F}} \eta\left[v_{h}-I_{h}^{k}\left(v_{h}\right)\right]\left[I_{h}^{k}\left(v_{h}\right)\right] d x \\
& =\int_{\Omega}\left(\sum_{i=0}^{d}\left(\alpha_{i}^{v_{h}}+T_{k}\left(\alpha_{i}^{v_{h}}\right)\right) A \nabla_{h} \varphi_{i}\right)\left(\sum_{j=0}^{d} T_{k}\left(\alpha_{j}^{v_{h}}\right)\right. \\
& \left.\cdot A \nabla_{h} \varphi_{j}\right) d x \\
& -\sum_{F \in \mathscr{F}_{h}^{i}} \int_{F}\left(\sum_{j=0}^{d} T_{k}\left(\alpha_{j}^{v_{h}}\right)\left[\varphi_{j}\right]\right) d x \\
& \\
& \left.\left.n_{i=0}^{v_{v_{h}}}+T_{k}\left(\alpha_{i}^{v_{h}}\right)\right)\left\{A \nabla_{h} \varphi_{i}\right\}_{\bar{\omega}}\right)
\end{aligned}
$$




$$
\begin{aligned}
& -\sum_{F \in \mathscr{F}_{h}^{i}} \int_{F}\left(\sum_{i=0}^{d} T_{k}\left(\alpha_{i}^{v_{h}}\right)\left\{\varphi_{i}\right\}_{\omega}\right) \\
& \cdot n_{F}\left(\sum_{j=0}^{d}\left(\alpha_{j}^{v_{h}}+T_{k}\left(\alpha_{j}^{v_{h}}\right)\right)\left[A \nabla_{h} \varphi_{j}\right]\right) d x \\
& +\sum_{F \in \mathscr{F}_{h}^{i}} \int_{F}\left(\sum_{i=0}^{d}\left(\alpha_{i}^{v_{h}}+T_{k}\left(\alpha_{i}^{v_{h}}\right)\right)\left[A \nabla_{h} \varphi_{i}\right]\right) \\
& \cdot\left(\sum_{j=0}^{d} T_{k}\left(\alpha_{j}^{v_{h}}\right)\left[\varphi_{j}\right]\right) d x=\sum_{i=0}^{d}\left(\alpha_{i}^{v_{h}}-T_{k}\left(\alpha_{i}^{v_{h}}\right)\right) \\
& \cdot \sum_{j=0}^{d} T_{k}\left(\alpha_{j}^{v_{h}}\right) a_{h}^{s w i p}\left(\varphi_{i}, \varphi_{j}\right)=\sum_{i=0}^{d}\left(\alpha_{i}^{v_{h}}+T_{k}\left(\alpha_{i}^{v_{h}}\right)\right) \\
& \cdot \sum_{j=0}^{d} T_{k}\left(\alpha_{j}^{v_{h}}\right) Q_{i, j}=\sum_{i=0}^{d} S_{i},
\end{aligned}
$$

where

$$
\begin{aligned}
S_{i}= & \left(\alpha_{i}^{v_{h}}-T_{k}\left(\alpha_{i}^{v_{h}}\right)\right) T_{k}\left(\alpha_{i}^{v_{h}}\right) Q_{i, i} \\
& +\left(\alpha_{i}^{v_{h}}-T_{k}\left(\alpha_{i}^{v_{h}}\right)\right) \sum_{\substack{0 \leq j \leq d \\
j \neq i}} \alpha_{j}^{T_{k}\left(v_{h}\right)} Q_{i, j} .
\end{aligned}
$$

Fix $\in\{0,1, \ldots, d\}$;

(i) if $\left|T_{k}\left(v_{h}\left(m_{i}\right)\right)\right|<k$, then $T_{k}\left(v_{h}\left(m_{i}\right)\right)=v_{h}\left(m_{i}\right)$ et $\alpha_{i}^{v_{h}}-$ $T_{k}\left(\alpha_{i}^{v_{h}}\right)=0$ and $S_{i}=0$

(ii) if $\left|T_{k}\left(v_{h}\left(m_{i}\right)\right)\right|=k$,

then

$$
\begin{aligned}
& \left(\alpha_{i}^{v_{h}}-T_{k}\left(\alpha_{i}^{v_{h}}\right)\right) T_{k}\left(\alpha_{i}^{v_{h}}\right) \\
& \quad=\left(v_{h}\left(m_{i}\right)-T_{k}\left(v_{h}\left(m_{i}\right)\right)\right) T_{k}\left(v_{h}\left(m_{i}\right)\right) \\
& \quad=k\left|v_{h}\left(m_{i}\right)-T_{k}\left(v_{h}\left(m_{i}\right)\right)\right|,
\end{aligned}
$$

and therefore

$$
S_{i} \geq k\left|v_{h}\left(m_{i}\right)-T_{k}\left(v_{h}\left(m_{i}\right)\right)\right|\left(Q_{i, i}-\sum_{\substack{0 \leq j \leq d \\ j \neq i}}\left|Q_{i, j}\right|\right)
$$

in the light of the assumption (32). This proves that $S_{i} \geq 0$ for every $i$ in $\{1,2, \ldots, N\}$; and so (117).

\section{Proof of the Main Theorem}

We first show an a priori estimate (compared with (50)) on the solution $u_{h}$ of (48).

Lemma 17. Let $v_{h} \in V_{h}, T \in \mathscr{T}_{h}$, and $k>0$ such that $\max _{m_{i} \in T}\left|v_{h}\right| \leq k$. Then

$$
\max _{T}\left|v_{h}\right| \leq k\left(2 d^{2}-1\right) .
$$

Proof. Let $v_{h} \in V_{h}, T \in \mathscr{T}_{h}$, and $k>0$ such that $\max _{m_{i} \in T}\left|v_{h}\right| \leq k$. For every $x \in T$,

$$
\begin{aligned}
\left|v_{h}(x)\right| & =\left|\sum_{i=0}^{d} \alpha_{i}^{v_{h}} \varphi_{i}(x)\right| \leq \sum_{i=0}^{d}\left|\alpha_{i}^{v_{h}} \varphi_{i}(x)\right| \\
& \leq \sum_{i=0}^{d}\left|v_{h}\left(m_{i}\right)\right|\left|\varphi_{i}(x)\right| \\
& \leq-k\left(\sum_{\substack{i=0 \\
\lambda_{i}<1 / d}}^{d} \varphi_{i}(x)\right)+k \underbrace{\left(1+2 \sum_{i=0}^{d}(d-1)\right) \leq k\left(2 d^{2}-1\right),}_{\left.=1-\sum_{\substack{d i=0 \\
\lambda_{i}<1 / d}}^{\sum_{\substack{i=0 \\
\lambda_{i} \geq 1 / d}}^{d} \varphi_{i}(x)}\right)}
\end{aligned}
$$

the inequality (123) is then proved.

Proposition 18. Under the assumption of Theorem 4, the solution $u_{h}$ of (48) satisfies for every $k>0$ and every $h>0$

$$
a_{h}^{\text {swip }}\left(I_{h}^{k}\left(u_{h}\right), I_{h}^{k}\left(u_{h}\right)\right) \leq \int_{\Omega} f I_{h}^{k}\left(u_{h}\right) d x,
$$

and, in particular, $u_{h}$ satisfies

$$
\int_{\Omega}\left|\nabla_{h} I_{h}^{k}\left(u_{h}\right)\right|^{2} d x \leq k \frac{2 d^{2}-1}{\alpha C_{\eta}}\|f\|_{L^{1}(\Omega)},
$$

where the constant $C_{\eta}$ only depends on $\eta, \sigma$, and $d$.

The proof makes use of results appearing in [3] that we reproduce as follows.

Lemma 19 (Lemma 1.46 in [3], discrete trace inequality). Under assumption (47), one has for all $v_{h} \in \mathbb{P}_{d}^{1}\left(\mathscr{T}_{h}\right)$, all $T$ $\in \mathscr{T}_{h}$, and all $F \in \mathscr{F}_{T}$

$$
h_{T}^{1 / 2}\left\|v_{h}\right\|_{L^{2}(F)} \leq C_{t r}\left\|v_{h}\right\|_{L^{2}(T)}
$$

where $C_{t r}$ only depends on $\sigma$ and $d$.

Lemma 20 (Lemma 4.51 in [3], discrete coercivity). For all $\eta>\eta:=(d+1) C_{t r}^{2}$, the SWIP bilinear form $a_{h}^{\text {swip }}$ is coercive on $\overline{V_{h}}$ with respect to the $\|\cdot\|_{\text {swip }}-$ norm; i.e.,

$$
\forall v_{h} \in V_{h}, \quad a_{h}^{\text {swip }}\left(v_{h}, v_{h}\right) \geq C_{\eta}\left\|v_{h}\right\|_{\text {swip }}^{2}
$$

where $C_{\eta}=\left(\eta-(d+1) C_{t r}^{2}\right) /(1+\eta)$.

Proof (of Proposition 18). Using $I_{h}^{k}\left(u_{h}\right)$ as a test function in (48) one has

$$
a_{h}^{\text {swip }}\left(u_{h}, I_{h}^{k}\left(u_{h}\right)\right)=\int_{\Omega} f I_{h}^{k}\left(u_{h}\right) d x,
$$

and from (117) we obtain (125). 
On the other hand, by combining (125) with coercivities (34) and (128) one obtains successively

$$
\begin{aligned}
& \alpha C_{\eta} \int_{\Omega}\left|\nabla_{h}\left(I_{h}^{k}\left(u_{h}\right)\right)\right|^{2} d x \\
& \quad \leq C_{\eta} \int_{\Omega}\left|A \nabla_{h}\left(I_{h}^{k}\left(u_{h}\right)\right) \nabla_{h}\left(I_{h}^{k}\left(u_{h}\right)\right)\right| d x \\
& \quad \leq C_{\eta}\left\|I_{h}^{k}\left(u_{h}\right)\right\|_{\text {swip }}^{2} \leq a_{h}^{\text {swip }}\left(I_{h}^{k}\left(u_{h}\right), I_{h}^{k}\left(u_{h}\right)\right) \\
& \quad \leq \int_{\Omega} f I_{h}^{k}\left(u_{h}\right) d x \leq \max _{\Omega}\left|I_{h}^{k}\left(u_{h}\right)\right| \int_{\Omega}|f| d x \\
& \quad \leq k\left(2 d^{2}-1\right)\|f\|_{L^{1}(\Omega)},
\end{aligned}
$$

and by (123) the estimation (126) is then proved.

Theorem 21. Under the assumptions of Theorem 4, the solution $u_{h}$ of (48) satisfies for every $q$ with $1 \leq q<d /(d-1)$

$$
\begin{aligned}
u_{h} & \longrightarrow u \quad \text { strongly in } L^{q}(\Omega), \\
\nabla_{h} u_{h} & \longrightarrow \nabla u \quad \text { strongly in }\left[L^{q}(\Omega)\right]^{d}, \\
\left|u_{h}\right|_{J, A, q} & \longrightarrow 0,
\end{aligned}
$$

when $h$ tends to zero, where $u$ is the unique renormalized solution of (1).

Proof (proof of Theorem 3.2 in [4]). Consider a sequence $f^{\varepsilon}$ in $L^{2}(\Omega)$, converging strongly in $L^{1}(\Omega)$ to $f$ (for example, $f^{\varepsilon}=$ $\left.T_{1 / \varepsilon}(f)\right)$. Let $u_{h}^{\varepsilon}$ to be the unique solution of (48) for the righthand side $f^{\varepsilon}$. Then $u_{h}-u_{h}^{\varepsilon}$ satisfies

$$
\begin{gathered}
u_{h}-u_{h}^{\varepsilon} \in V_{h}, \\
\forall v_{h} \in V_{h}, \\
a_{h}^{\text {swip }}\left(u_{h}-u_{h}^{\epsilon}, v_{h}\right)=\int_{\Omega}\left(f-f^{\epsilon}\right) v_{h} d x .
\end{gathered}
$$

Applying estimate (126) to this problem, we obtain for every $k>0$, every $h>0$, and every $\varepsilon>0$

$$
\begin{aligned}
& \int_{\Omega}\left|\nabla_{h}\left(I_{h}^{k}\left(u_{h}-u_{h}^{\epsilon}\right)\right)\right|^{2} d x \\
& \leq k \frac{2 d^{2}-1}{\alpha C_{\eta}}\left\|f-f^{\epsilon}\right\|_{L^{1}(\Omega)},
\end{aligned}
$$

Which implies by Theorem 5 that for every $q$ with $1 \leq q<$ $d /(d-1)$, every $h>0$, and every $\varepsilon>0$

$$
\begin{aligned}
& \left\|u_{h}-u_{h}^{\epsilon}\right\|_{\text {swip, } q} \\
& \leq \frac{C_{2}\left(d,|\Omega|, q, \sigma,\|A\|_{L^{\infty}(\Omega)^{d \times d}}\right)}{\alpha C_{\eta}}\left\|f-f^{\epsilon}\right\|_{L^{1}(\Omega)} .
\end{aligned}
$$

On the other hand, since $f^{\mathcal{E}} \in L^{2}(\Omega)$ and $\mathscr{T}_{h}$ satisfies (44), (46), and (47), it is known (see [3]) that for every fixed $\varepsilon$

$$
\begin{aligned}
u_{h}^{\varepsilon} & \longrightarrow u^{\varepsilon} \quad \text { strongly in } L^{2}(\Omega), \\
\nabla_{h} u_{h}^{\varepsilon} & \longrightarrow \nabla u^{\varepsilon} \quad \text { strongly in }\left[L^{2}(\Omega)\right]^{d}, \\
\left|u_{h}^{\varepsilon}\right|_{J, A} & \longrightarrow 0,
\end{aligned}
$$

when $h$ tends to zero, where $u^{\varepsilon}$ is the unique solution of

$$
\begin{gathered}
u^{\varepsilon} \in H_{0}^{1}(\Omega), \\
-\operatorname{div}\left(A \nabla u^{\varepsilon}\right)=f^{\varepsilon} \quad \text { in } \mathscr{D}^{\prime}(\Omega) .
\end{gathered}
$$

Finally, the function $u^{\varepsilon}$ is also the unique renormalized solution of the problem

$$
\begin{aligned}
-\operatorname{div}\left(A \nabla u^{\varepsilon}\right)=f^{\varepsilon} & \text { in } \Omega, \\
u^{\varepsilon}=0 & \text { on } \partial \Omega .
\end{aligned}
$$

The estimate (43) combined with the inequality

$$
\left\|u^{\epsilon}-u\right\|_{\text {swip,q }} \leq\|A\|_{L^{\infty}(\Omega) d \times d}\left\|u^{\epsilon}-u\right\|_{W_{0}^{1, q}(\Omega)}
$$

allows one to have

$$
\begin{aligned}
& \left\|u^{\epsilon}-u\right\|_{\text {swip, } q} \\
& \quad \leq \frac{\|A\|_{L^{\infty}(\Omega)^{d \times d}} C_{1}(d,|\Omega|, q)}{\alpha}\left\|f-f^{\epsilon}\right\|_{L^{1}(\Omega)},
\end{aligned}
$$

for every $q$ with $1 \leq q<d /(d-1)$, where $u$ is the unique renormalized solution of (1).

Writing now

$$
\begin{aligned}
& \left\|u_{h}-u\right\|_{\text {swip,q }} \leq\left\|u_{h}-u_{h}^{\epsilon}\right\|_{\text {swip,q }}+\left\|u^{\epsilon}-u\right\|_{\text {swip,q }} \\
& +\left\|u_{h}^{\epsilon}-u^{\epsilon}\right\|_{s w i p, q},
\end{aligned}
$$

Using (134), (136), (137), and (141), one has for every $\varepsilon>0$ and every $q$ with $1 \leq q<d /(d-1)$

$$
\begin{aligned}
& \limsup _{h \rightarrow 0}\left\|u_{h}-u\right\|_{\text {swip, } q} \\
& \quad \leq \frac{C_{3}\left(d,|\Omega|, q, \sigma,\|A\|_{L^{\infty}(\Omega)^{d \times d}}\right)}{\alpha C_{\eta}}\left\|f-f^{\epsilon}\right\|_{L^{1}(\Omega)},
\end{aligned}
$$

and passing to the limit when $\varepsilon$ tends to zero proves Theorem 21.

To complete the proof of Theorem 4, it remains to prove the following proposition.

Proposition 22. Under the assumptions of Theorem 4, the solution $u_{h}$ of (48) satisfies

$$
\begin{gathered}
I_{h}^{k}\left(u_{h}\right) \underset{h \rightarrow 0}{\longrightarrow} T_{k}(u) \quad \text { strongly in } L^{2}(\Omega), \\
\nabla_{h}\left(I_{h}^{k}\left(u_{h}\right)\right) \underset{h \rightarrow 0}{\longrightarrow} \nabla T_{k}(u) \quad \text { strongly in }\left[L^{2}(\Omega)\right]^{d}, \\
\left|I_{h}^{k}\left(u_{h}\right)\right|_{J, A} \underset{h \rightarrow 0}{\longrightarrow} 0,
\end{gathered}
$$

for every $k>0$. 
Proof (proof of Proposition 3.3 in [4]). First by result of Proposition 15 and the estimate (126) one can have (see theorem 5.7 in [3]) the two following convergences:

$$
\begin{gathered}
I_{h}^{k}\left(u_{h}\right) \underset{h \rightarrow 0}{\longrightarrow} T_{k}(u) \quad \text { strongly in } L^{2}(\Omega), \\
G_{h}^{l}\left(I_{h}^{k}\left(u_{h}\right)\right) \underset{h \rightarrow 0}{\longrightarrow} \nabla T_{k}(u) \quad \text { weakly in }\left[L^{2}(\Omega)\right]^{d},
\end{gathered}
$$

for every $k>0$

On the other hand, using (123) one has

$$
\left|f I_{h}^{k}\left(u_{h}\right)\right| \leq k\left(2 d^{2}-1\right)|f| \in L^{1}(\Omega),
$$

so, by Lebesgue's dominated convergence theorem combined with discrete Rellich-Kondrachov's compactness theorem (see theorem 5.6 in [3]) one has

$$
\int_{\Omega} f I_{h}^{k}\left(u_{h}\right) \mathrm{d} x \underset{h \rightarrow 0}{\longrightarrow} \int_{\Omega} f T_{k}(u) \mathrm{d} x .
$$

Therefore passing to the limit with respect to $h$ in (125) yields

$$
\limsup _{h \rightarrow 0} a_{h}^{\text {swip }}\left(I_{h}^{k}\left(u_{h}\right), I_{h}^{k}\left(u_{h}\right)\right) \leq \int_{\Omega} f T_{k}(u) d x .
$$

Consequently

$$
\begin{aligned}
& \limsup _{h \rightarrow 0} \int_{\Omega} A G_{h}^{l}\left(I_{h}^{k}\left(u_{h}\right)\right) G_{h}^{l}\left(I_{h}^{k}\left(u_{h}\right)\right) \\
& \quad \leq \int_{\Omega} f T_{k}(u) d x .
\end{aligned}
$$

By the fact that

$$
\begin{gathered}
\int_{\Omega} A G_{h}^{l}\left(I_{h}^{k}\left(u_{h}\right)\right) G_{h}^{l}\left(I_{h}^{k}\left(u_{h}\right)\right) \\
\leq a_{h}^{\operatorname{swip}}\left(I_{h}^{k}\left(u_{h}\right), I_{h}^{k}\left(u_{h}\right)\right),
\end{gathered}
$$

and since $u$ is the renormalized solution of (1), it is known that (see [4])

$$
\int_{\Omega} A \nabla T_{k}(u) \nabla T_{k}(u)=\int_{\Omega} f T_{k}(u) d x .
$$

Finally, from (152) and (154), we deduce that

$$
\begin{aligned}
& \underset{h \rightarrow 0}{\limsup } \int_{\Omega} A G_{h}^{l}\left(I_{h}^{k}\left(u_{h}\right)\right) G_{h}^{l}\left(I_{h}^{k}\left(u_{h}\right)\right) \\
& \quad \leq \int_{\Omega} A \nabla T_{k}(u) \nabla T_{k}(u),
\end{aligned}
$$

which combined with the weak convergence (148) implies

$$
G_{h}^{l}\left(I_{h}^{k}\left(u_{h}\right)\right) \underset{h \rightarrow 0}{\longrightarrow} \nabla T_{k}(u) \quad \text { strongly in }\left[L^{2}(\Omega)\right]^{d} \text {. }
$$

Owing to Proposition 4.36 in [3], for all $v_{h} \in V_{h}$ and all $\eta>(d+1) C_{t r}^{2}$, one can have

$$
\begin{aligned}
& \left|I_{h}^{k}\left(u_{h}\right)\right|_{J, A} \\
& \leq \frac{a_{h}^{\text {swip }}\left(I_{h}^{k}\left(u_{h}\right), I_{h}^{k}\left(u_{h}\right)\right)-\left\|A^{1 / 2} G_{h}^{l}\left(I_{h}^{k}\left(u_{h}\right)\right)\right\|_{\left[L^{2}(\Omega)\right]^{d}}}{\eta-(d+1) C_{t r}^{2}}
\end{aligned}
$$

and, since the right-hand side tends to zero, the result (146) holds.

Finally, using the result of Proposition 4.34 in [3]

$$
\left\|R_{h}^{l}\left(\left[I_{h}^{k}\left(u_{h}\right)\right]\right)\right\|_{\left[L^{2}(\Omega)\right]^{d}} \leq \sqrt{d+1} C_{t r}\left|I_{h}^{k}\left(u_{h}\right)\right|_{J, A}
$$

with the triangle inequality that yields

$$
\begin{aligned}
& \left\|\nabla_{h} I_{h}^{k}\left(u_{h}\right)-\nabla T_{k}(u)\right\|_{\left[L^{2}(\Omega)\right]^{d}} \\
& \leq\left\|G_{h}^{l}\left(I_{h}^{k}\left(u_{h}\right)\right)-\nabla T_{k}(u)\right\|_{\left[L^{2}(\Omega)\right]^{d}} \\
& \quad+\left\|R_{h}^{l}\left(\left[I_{h}^{k}\left(u_{h}\right)\right]\right)\right\|_{\left[L^{2}(\Omega)\right]^{d}}
\end{aligned}
$$

concluding the proof of (145).

\section{The Case Where f Is a Bounded Radon Measure}

The materials used in [4], to handle the case where $\mathrm{f}$ belongs to $\mathscr{M}_{b}(\Omega)$, are not specific to the case of $\mathbb{P}_{1}$ finite elements approximation; only the weak convergence (148) requires clarification; in our approach it is based on the result of Proposition 15 whose proof involves only properties of $v_{h}$ not $f$. So we can also state the following convergence result.

Theorem 23 (Theorem 4.1 in [4]). Assume that $f$ belongs to $\mathscr{M}_{b}(\Omega)$ and $A$ and $\mathscr{T}_{h}$ satisfy (46), (33), (35), (44), and (47) and (32). Then there exist a subsequence, still denoted by $h$, and a function $u$ such that for every $k>0$ and for every $q$ with 1 $\leq q<d /(d-1)$ one has

$$
\begin{gathered}
G_{h}^{l}\left(I_{h}^{k}\left(u_{h}\right)\right) \rightarrow \nabla T_{k}(u) \quad \text { weakly in }\left[L^{2}(\Omega)\right]^{d}, \\
\nabla_{h} u_{h} \rightarrow \nabla u \text { weakly in }\left[L^{q}(\Omega)\right]^{d},
\end{gathered}
$$

when $h$ tends to zero along this subsequence, where $u$ satisfies

$$
\forall k>0, \quad T_{k}(u) \in H_{0}^{1}(\Omega),
$$

$$
\begin{aligned}
& \forall q \text { with } 1 \leq q<\frac{d}{d-1}, \quad u \in W_{0}^{1, q}(\Omega) \\
& \forall v \in \mathscr{C}_{c}^{\infty}(\Omega), \quad \int_{\Omega} A \nabla u \nabla v d x=\int_{\Omega} v d f .
\end{aligned}
$$

\section{Convergence Rate Estimation}

\subsection{Error Estimates for Smooth Solutions}

Assumption 24 (regularity of exact solution and space $V_{*}$ ). As in [3], we assume that $\mathscr{T}_{h}$ is compatible with the partition $P_{\Omega}$ 
in the sense of Assumption 3, and the unique solution $v$ is such that

$$
v \in V_{*}:=H_{0}^{1}(\Omega) \cap H^{2}\left(\mathscr{T}_{h}\right) .
$$

And we set

$$
V_{* h}:=V_{*}+V_{h}
$$

The convergence analysis is performed in the spirit of (Theorem 1.35 [3]) by establishing discrete coercivity, consistency, and boundedness for $a_{h}^{\text {swip }}$. The discrete bilinear form $a_{h}^{\text {swip }}$ is extended to $V_{* h} \times V_{h}$.

Without further knowledge on the exact solution v apart from the domain $\Omega$ and the datum $g \in L^{2}(\Omega)$, Assumption 24 can be asserted for instance if the domain $\Omega$ is convex; see Grisvard [7].

A straightforward consequence of the Lax-Milgram Lemma is that the discrete problem (48) is well-posed.

Theorem 25. Under the assumptions of Theorem $4(d=2$ or $d=3$ ), iff belongs to $L^{r, \infty}(\Omega)$ for some $r$ such that $1<r<$ 2, $\Omega$ a convex polyhedron $\left(\Omega_{h}=\Omega\right)$, and $A \in\left[W^{1, \infty}(\Omega)\right]^{d \times d}$, then $\forall q$ with $1 \leq q<d /(d-1)$

$$
\begin{aligned}
& \left\|u_{h}-u\right\|_{\text {swip,q }} \leq C\left(d,|\Omega|, q, r, \alpha,\|A\|_{W^{1, \infty}(\Omega)^{d \times d}}, \sigma\right) \\
& \cdot h^{2(1-1 / r)}\|f\|_{L^{r, \infty}(\Omega)} .
\end{aligned}
$$

Proof (proof of Theorem 5.1 in [4]). From [3] the unique solution $u^{\epsilon}$ of (48) with right-hand side $f^{\epsilon}=T_{1 / \epsilon}(f) \epsilon$ $L^{\infty}(\Omega) \subset L^{2}(\Omega)$ verifies

$$
\left\|u_{h}^{\epsilon}-u^{\epsilon}\right\|_{\text {swip }} \leq C\left(\|A\|_{W^{1, \infty}(\Omega)^{d \times d}}\right) h\left\|f^{\epsilon}\right\|_{L^{2}(\Omega)}
$$

so

$$
\begin{aligned}
& \left\|u_{h}^{\epsilon}-u^{\epsilon}\right\|_{\text {swip,q }} \\
& \quad \leq C\left(q, d, \eta,|\Omega|,\|A\|_{W^{1, \infty}(\Omega)^{d \times d}}\right) h\left\|f^{\epsilon}\right\|_{L^{2}(\Omega)}
\end{aligned}
$$

Combined with (142), (134), and (141) allows one to have

$$
\begin{aligned}
\left\|u_{h}-u\right\|_{\text {swip }, q} \leq & C\left(q, d, \eta,|\Omega|,\|A\|_{W^{1, \infty}(\Omega)^{d \times d}}\right) \\
& \cdot\left(\left\|f-f^{\epsilon}\right\|_{L^{1}(\Omega)}+h\left\|f^{\epsilon}\right\|_{L^{2}(\Omega)}\right),
\end{aligned}
$$

and by proceeding as in [4], we obtain (173).

Remark 26. If $f \in L^{r, \infty}(\Omega)$ for some $r$ with $1<r<2$ and

$$
\forall k>0: \quad T_{k}(f) \in H^{1}(\Omega),
$$

then a small adaptation of the proof given in [4] provides an $O\left(h^{4(1-1 / r)}\right)$ error estimate in \|\|$_{\text {swip, } q}$-norm, with $1 \leq q<$ $d /(d-1)$, since, with (168), it is known that

$$
\begin{aligned}
& \left\|u_{h}^{\epsilon}-u^{\epsilon}\right\|_{\text {swip }, q} \\
& \quad \leq C\left(d,|\Omega|, q, r, \alpha,\|A\|_{W^{1, \infty}(\Omega)^{d \times d}}, \sigma\right) h^{2}\left\|f^{\epsilon}\right\|_{L^{2}(\Omega)} .
\end{aligned}
$$

\subsection{Error Estimates for Low-Regularity Solutions}

Assumption 27 (regularity of exact solution and space $V_{*}$ ). As in [3], we assume that the mesh $\mathscr{T}_{h}$ is compatible with the partition $P_{\Omega}$ in the sense of Assumption 3, $d \geq 2$, and that there is $p$ such that $2 d /(d+2)<p \leq 2$; the unique solution $v$ is such that

$$
v \in V_{*}:=H_{0}^{1}(\Omega) \cap W^{2, p}\left(\mathscr{T}_{h}\right),
$$

where $W^{2, p}\left(P_{\Omega}\right)=W^{2, p}(\mathscr{T})$ designate that the mesh $\mathscr{T}$ is compatible with the partition $P_{\Omega}$, and we set

$$
V_{* h}:=V_{*}+V_{h}
$$

We also assume $p<2$ since, in the case $p=2$, Assumption 27 amounts to Assumption 24.

Assumption 27 requires $\mathrm{p}>1$ for $\mathrm{d}=2$ and $\mathrm{p}>6 / 5$ for $\mathrm{d}=$ 3. In particular, we observe that, in two space dimensions, $v \in$ $W^{2, p}\left(P_{\Omega}\right)$ with $\mathrm{p}>1$ holds true in polygonal domains; see, e.g., Dauge [8]. Moreover, using Sobolev embeddings (see [Evans [9], Sect. 5.6] or [Brézis [10], Sect. IX.3]), Assumption 27 implies

$$
v \in H^{1+\alpha_{p}}(\Omega), \quad \text { with } \alpha_{p}=\frac{d+2}{2}-\frac{d}{p}>0 .
$$

Theorem 28. Under the assumptions of Theorem $4(d=2$ or $d=3$ ), iff belongs to $L^{r, \infty}(\Omega)$ for some $r$ such that $1<r<2$, $\Omega$ a convex polyhedron $\left(\Omega_{h}=\Omega\right)$, and $A \in\left[W^{1, \infty}(\Omega)\right]^{d \times d}$, then $\forall q$ with $1 \leq q<p$

$$
\begin{gathered}
\left\|u_{h}-u\right\|_{\text {swip, } q} \leq C\left(d,|\Omega|, q, r, \alpha,\|A\|_{W^{1, \infty}(\Omega)^{d \times d}}, \sigma\right) \\
\cdot h^{2 \alpha_{p}(1-1 / r)}\|f\|_{L^{r, \infty}(\Omega)} .
\end{gathered}
$$

Remark 29. Moreover, under the assumption in Remark 26 one can have an $O\left(h^{4 \alpha_{p}(1-1 / r)}\right)$ error estimate in \|\|$_{\text {swip, } q}$-norm, with $1 \leq q<p$.

\section{The Case Where A Is the Identity Matrix}

Returning to the definition (31) of mass matrix $Q$ where $A=$ $I$, one has 
for $i, j$ in $\{1,2, \ldots, N\}$

$$
\begin{aligned}
Q_{i, j} & =a_{h}^{\text {swip }}\left(\varphi_{i}, \varphi_{j}\right) \\
& =\underbrace{\int_{\Omega} \nabla_{h} \varphi_{i} \nabla_{h} \varphi_{j} d x}_{=I_{1}}+\underbrace{\sum_{F \in \mathscr{F}_{h}^{i}} \frac{1}{h_{F}} \eta\left[\varphi_{i}\right]\left[\varphi_{j}\right] d x}_{=I_{2}}-\underbrace{\left(\sum_{F \in \mathscr{F}_{h}^{i}} \int_{F}\left\{\nabla_{h} \varphi_{i}\right\}_{\omega} \cdot n_{F}\left[\varphi_{j}\right] d x+\sum_{F \in \mathscr{F}_{h}^{i}} \int_{F}\left\{\nabla_{h} \varphi_{j}\right\}_{\omega} \cdot n_{F}\left[\varphi_{i}\right] d x\right)}_{=I_{3}} .
\end{aligned}
$$

If $i=j$, so

$$
\begin{aligned}
I_{1} & =\int_{\Omega} \nabla_{h} \varphi_{i}^{2} d x \geq 0, \\
I_{2} & =\sum_{F \in \mathscr{F}_{h}^{i}} \int_{F} \frac{\eta}{h_{F}}\left[\varphi_{i}\right]^{2} d x \geq 0, \\
I_{3} & =2\left(\int_{F_{i}}\left\{\nabla_{h} \varphi_{i}\right\}_{\omega} \cdot n_{F}\left[\varphi_{i}\right] d x\right. \\
& \left.+\sum_{\substack{F \in \mathscr{F}^{i} \\
F \neq F_{i}}}\left\{\nabla_{h} \varphi_{i}\right\}_{\omega} \cdot n_{F}\left[\varphi_{i}\right] d x\right)=0,
\end{aligned}
$$

since $\left[\varphi_{i}\right]_{F_{i}}=0,\left\{\nabla_{h} \varphi_{i}\right\}_{\omega} \cdot n_{F}=-\left(d / h_{F_{i}}\right) n_{i} \cdot n_{F}$, and $\int_{F \neq F_{i}} \varphi_{i} d x=0$.

$$
\text { If } i \neq j \text {, so }
$$

$$
I_{1}=\int_{\Omega} \nabla_{h} \varphi_{i} \nabla_{h} \varphi_{j} d x=\frac{1}{d^{2}}\left|F_{i}\right|\left|F_{j}\right| n_{i} \cdot n_{j} \leq 0
$$

(see Proposition 6.1 in [4]),

$$
\begin{aligned}
I_{2} & =\int_{F \in F_{i} \cup F_{j}} \frac{\eta}{h_{F}}\left[\varphi_{i}\right]\left[\varphi_{j}\right] d x+\sum_{\substack{F \in \mathscr{F}_{h} \\
F \neq F_{i} \neq F_{j}}} \frac{\eta}{h_{F}}\left[\varphi_{i}\right]\left[\varphi_{j}\right] d x \\
& =-\frac{\eta}{d+1} \sum_{\substack{F \in \mathscr{F}_{h}^{i} \\
F \neq F_{i} \neq F_{j}}} \frac{|F|}{h_{F}} \leq 0, \\
I_{3} & =-d \sum_{\substack{F \in \mathscr{F}_{h}^{i} \\
F \neq F_{i} \neq F_{j}}}\left(\frac{1}{h_{F_{i}}} n_{i} \cdot n_{F} \varphi_{j}+\frac{1}{h_{F_{j}}} n_{j} \cdot n_{F} \varphi_{i}\right) d x=0 .
\end{aligned}
$$

It is therefore concluded that, under the condition $n_{l} \cdot n_{k} \leq$ $0(l \neq k)$, one can have $Q_{l k} \leq 0$. Thus, the matrix $Q$ verifies (32).

\section{Data Availability}

No data were used to support this study.

\section{Conflicts of Interest}

The authors declare that they have no conflicts of interest.

\section{References}

[1] G. Dal Maso, F. Murat, L. Orsina, and A. Prignet, "Renormalized solutions of elliptic equations with general measure data," Annali della Scuola Normale Superiore di Pisa. Classe di Scienze. Serie IV, vol. 28, no. 4, pp. 741-808, 1999.

[2] P. Bénilan, L. Boccardo, T. Gallouët, R. Gariepy, M. Pierre, and J. L. Vázquez, "An L1-theory of existence and uniqueness of solutions of nonlinear elliptic equations," Ann. Scuola Norm. Sup. Pisa, vol. 22, no. 2, pp. 241-273, 1995.

[3] D. A. Di Pietro and A. Ern, "Mathematical aspects of discontinuous Galerkin methods," Mathématiques et Applications, vol. 69, 2011.

[4] J. Casado-Daz, T. Chacón Rebollo, V. Girault, M. Gómez Mármol, and F. Murat, "Finite elements approximation of second order linear elliptic equations in divergence form with right-hand side in L1," Numerische Mathematik, vol. 105, no. 3, pp. 337-374, 2007.

[5] L. Boccardo and T. Gallouët, "Nonlinear elliptic and parabolic equations involving measure data," Journal of Functional Analysis, vol. 87, no. 1, pp. 149-169, 1989.

[6] P. G. Ciarlet, The Finite Element Method for Elliptic Problems, North-Holland, Amsterdam, The Netherlands, 1978.

[7] P. Grisvard, Singularities in Boundary Value Problems, Masson, Paris, 1992.

[8] M. Dauge, Elliptic Boundary Value Problems on Corner Domains, vol. 1341 of Lecture Notes in Mathematics, Springer, Berlin, Germany, 1988.

[9] L. C. Evans, Partial Differential Equations, vol. 19 of Graduate Studies in Mathematics, American Mathematical Society, Providence, RI, USA, 1998.

[10] H. Brezis, Analyse Fonctionnelle, Masson, Paris, France, 1983. 


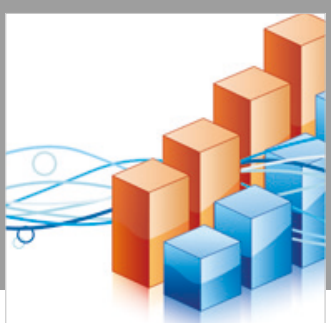

Advances in

Operations Research

\section{-n-m}
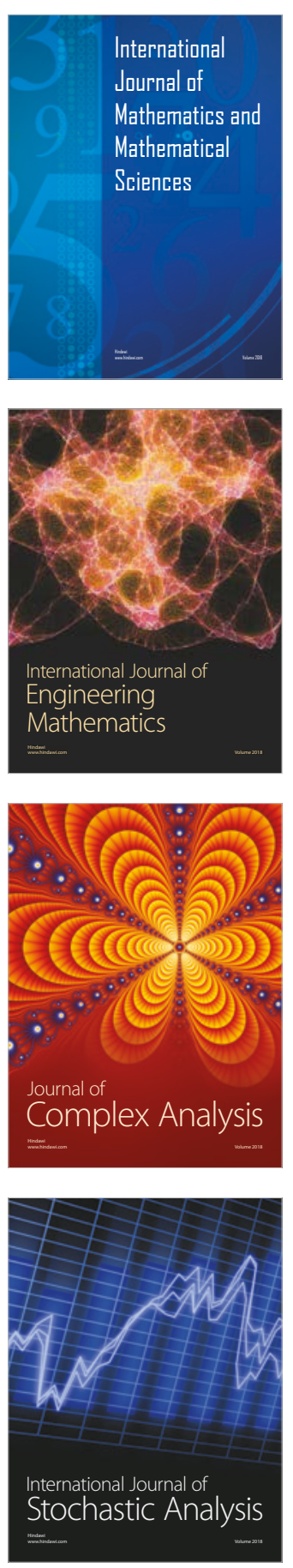
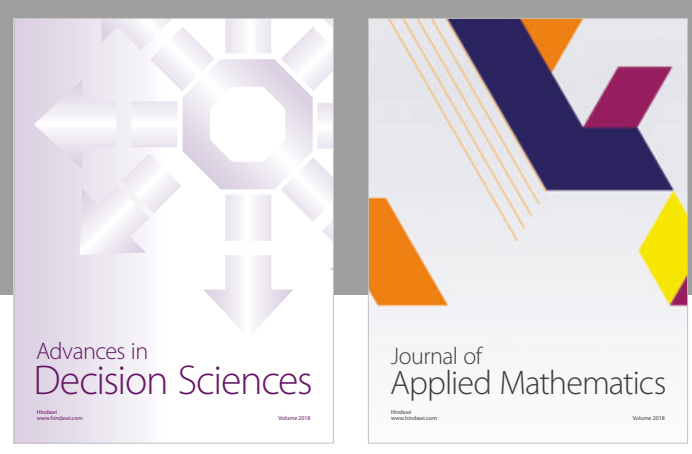

Journal of

Applied Mathematics
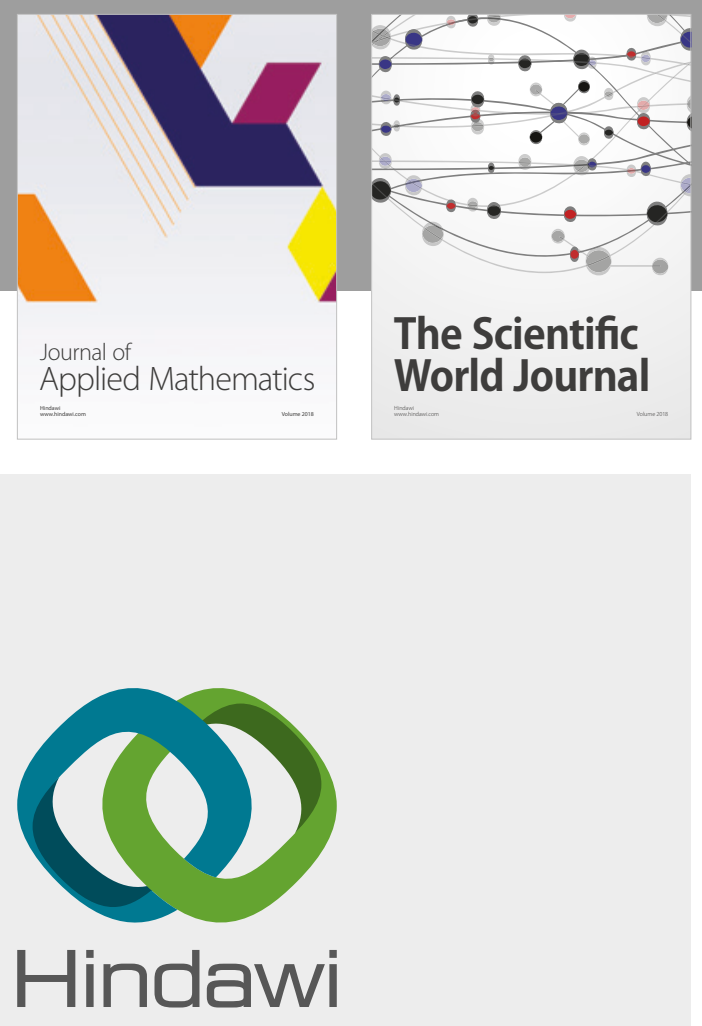

Submit your manuscripts at

www.hindawi.com

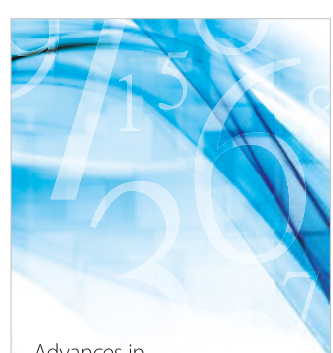

Advances in
Numerical Analysis
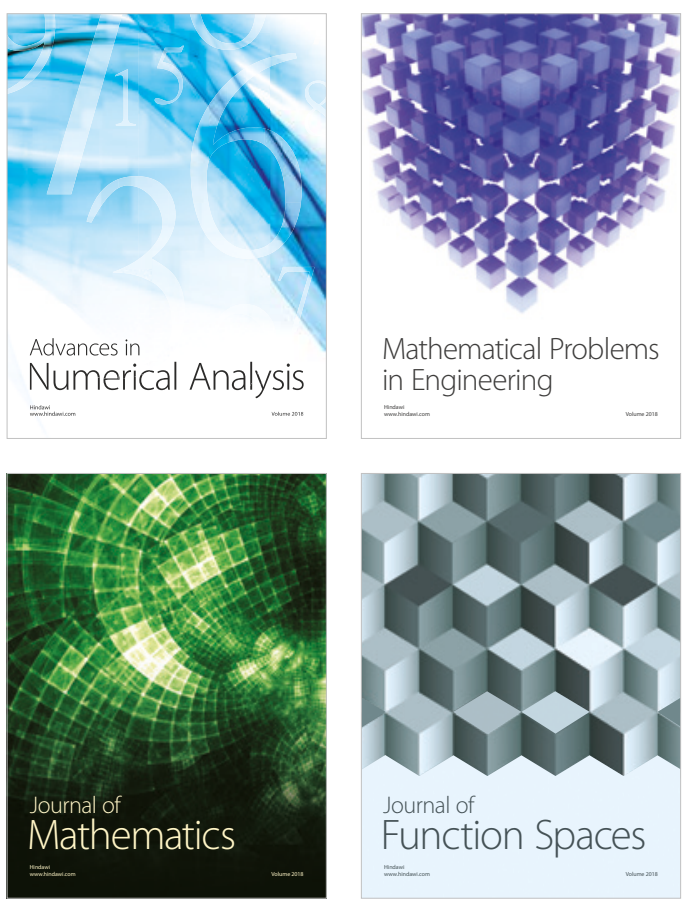

Mathematical Problems in Engineering

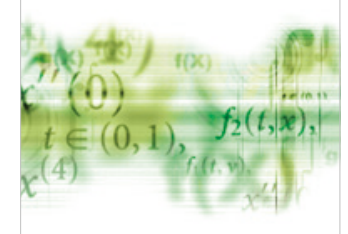

International Journal of

Differential Equations

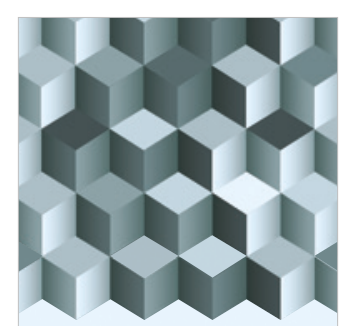

Journal of

Function Spaces

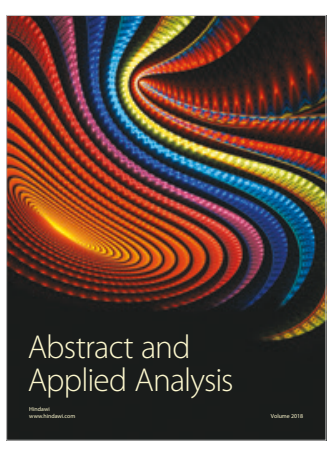

The Scientific

World Journal

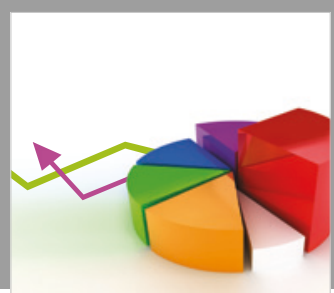

Journal of

Probability and Statistics
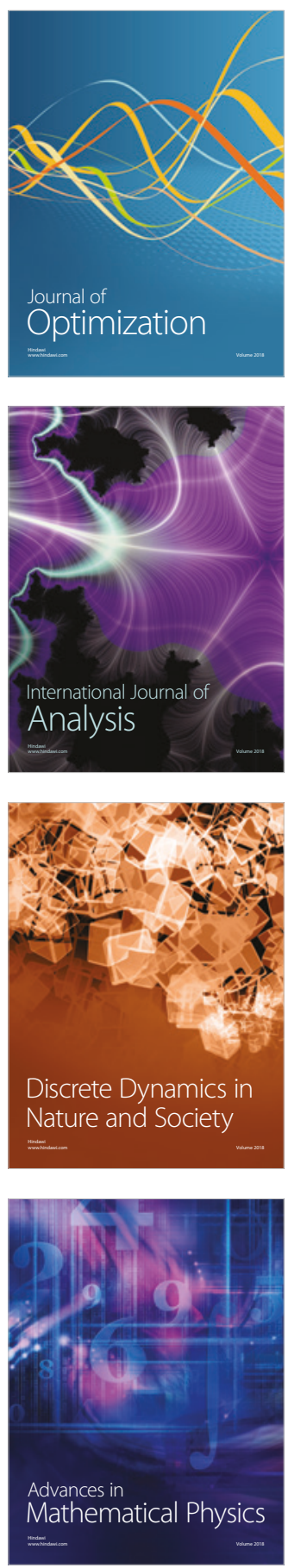